\title{
Fault Detection for Fuzzy Systems With Intermittent Measurements
}

\author{
Yan Zhao, James Lam, Senior Member, IEEE, and Huijun Gao, Member, IEEE
}

\begin{abstract}
This paper investigates the problem of fault detection for Takagi-Sugeno (T-S) fuzzy systems with intermittent measurements. The communication links between the plant and the fault detection filter are assumed to be imperfect (i.e., data packet dropouts occur intermittently, which appear typically in a network environment), and a stochastic variable satisfying the Bernoulli random binary distribution is utilized to model the unreliable communication links. The aim is to design a fuzzy fault detection filter such that, for all data missing conditions, the residual system is stochastically stable and preserves a guaranteed performance. The problem is solved through a basis-dependent Lyapunov function method, which is less conservative than the quadratic approach. The results are also extended to T-S fuzzy systems with time-varying parameter uncertainties. All the results are formulated in the form of linear matrix inequalities, which can be readily solved via standard numerical software. Two examples are provided to illustrate the usefulness and applicability of the developed theoretical results.
\end{abstract}

Index Terms-Basis-dependent Lyapunov functions, fault detection, intermittent measurements, Takagi-Sugeno (T-S) fuzzy systems, uncertainties.

\section{INTRODUCTION}

I $\mathrm{N}$ CONTROL systems, due to the unexpected variations in external surroundings, normal wear in components, or sudden changes in signals, there may appear different kinds of malfunction or imperfect behavior in normal operations, and people call them faults. Since a fault can degrade a system's performance and even cause catastrophic accidents, it is of great significance to detect it in time for the safety and reliability of control systems. The objective of fault detection is to detect the fault signal accurately whenever it appears. Many researchers have devoted themselves to investigating this problem, and a lot of methods have been established, mainly including the modelbased fault detection approach [2], [4], the parameter estimation approach [24], and the generalized likelihood method [30]. Among these methods, the model-based one is very popular, which is to design a fault detection filter or observer generating a residual including a threshold to detect the fault signal. In virtue

Manuscript received April 15, 2008; revised August 26, 2008 and November 5, 2008; accepted December 24, 2008. First published February 10, 2009; current version published April 1, 2009. This work was supported in part by the National Natural Science Foundation of China (60825303, 60834003), in part by the 973 Project (2009CB320600), in part by the Research Fund for the Doctoral Programme of Higher Education of China (20070213084), in part by the Heilongjiang Outstanding Youth Science Fund (JC200809), and in part by Postdoctoral Science Foundation of China (200801282), and in part by the University of Hong Kong Research and Conference Grants (CRCG) under Grant 200707176077.

Y. Zhao and H. Gao are with the Space Control and Inertial Technology Research Center, Harbin Institute of Technology, Harbin 150001, China (email: zhaoresponsible@gmail.com; hjgao@hit.edu.cn).

J. Lam is with the Department of Mechanical Engineering, University of Hong Kong, Hong Kong (e-mail: james.lam@hku.hk).

Digital Object Identifier 10.1109/TFUZZ.2009.2014860 of the advancement of modeling and state estimation techniques [6], [7], [19], [20], model-based fault detection has been well developed [26]. To mention a few, fault detection problems have been investigated for sampled-data systems in [9] and [32], uncertain systems in [1], [11], and [33], systems with time delays in [10], and Markovian jump linear systems in [14].

Most of the aforementioned results are concerned with linear models. But in reality, most physical systems are nonlinear, and thus, how to develop effective fault detection methods for nonlinear systems is an important and practical problem. However, the difficulty in modeling nonlinearities makes fault detection a hard task. To solve this problem, some researchers model the nonlinear plants as differential equations and solve the fault detection problem based on the conventional nonlinear system theory [16], whose limitations often do not generalize the obtained results. Other researchers take the advantage of artificial intelligence techniques [23], and use the conventional fuzzy models to represent the nonlinear systems by applying the inference engine. It is worth mentioning that there are no systematic and consistent approaches for the stability and performance analysis of those conventional fuzzy systems, and hence, the applicability of those results is also limited.

In recent years, Takagi-Sugeno (T-S) fuzzy models are playing more and more important roles in dealing with problems concerning nonlinear systems [3]. It has been proven that T-S fuzzy systems with affine terms can smoothly approximate any nonlinear functions to any specified accuracy within any compact set, which provides a theoretical foundation for using $\mathrm{T}-\mathrm{S}$ fuzzy models to represent complex nonlinear systems approximately. Meanwhile, T-S fuzzy models formulate the complex nonlinear systems into a framework that interpolates some affine local models by a set of fuzzy membership functions. Based on this framework, a systematic analysis and design procedure for complex nonlinear systems can be possibly developed in view of the powerful control theories and techniques in linear systems. The T-S fuzzy model has attracted great interests from researchers, and a number of results have been reported in literatures, including stability analysis [13], [27], stabilizing and $H_{\infty}$ control design [12], [21], [34], and state estimation [35]. Since T-S fuzzy models have provided a convenient way to study nonlinear systems, a feasible solution of the fault detection problem for nonlinear systems can be converted to that of fault detection for T-S fuzzy systems [17].

On the other hand, data packet dropout phenomena may often appear in many practical situations, i.e., some measurements or control inputs may be lost during the transmission. This problem has attracted more and more attention as the insertion of networked control systems (NCSs) in the control loops becomes popular [15]. Compared with the traditional point-topoint communication bus, NCSs have several advantages such 
as low cost, reduced weight and power requirements, simple installation and maintenance, and high reliability. However, in an NCS, since several components communicate over a shared network, information flows are prone to the curse of time sharing, and data loss always inevitably occurs from the plant to the filter or controller. Since data packet dropout can degrade a system's performance and even cause instability, it has been regarded as an important issue in the analysis and synthesis of network-based control systems, and some researchers have begun to study various problems of control systems, simultaneously considering this communication issue [28]. It is noted that most investigations concerning the data loss phenomenon are focused on the stability analysis and synthesis, and the plants are mostly linear. To the best of the our knowledge, there are no results about the fault detection problem for nonlinear systems with intermittent measurements.

Motivated by the aforementioned observations, in this paper, we investigate the problem of fault detection for T-S fuzzy systems with intermittent measurements. The measurements between the plant and the fault detection filter are assumed to be intermittent, and a stochastic variable is utilized to describe the imperfect communication links. Attention is focused on the fuzzy fault detection filter design such that the residual system is stochastically stable with the prescribed performance. A basis-dependent Lyapunov function is utilized in the derivative process, which renders the results to be potentially less conservative. Furthermore, the results are extended to T-S fuzzy systems with time-varying uncertainties. All the results are formulated in the form of linear matrix inequalities (LMIs). Two examples are illustrated to show the usefulness and applicability of the obtained results.

The remainder of the paper is organized as follows. Section II formulates the problem under consideration. Section III presents the fault detection filter design for the nominal fuzzy system, and the results are extended to the fuzzy system with timevarying uncertainties in Section IV. Two examples are illustrated in Section $\mathrm{V}$ to show the usefulness and applicability of the proposed approaches, and the paper is concluded in Section VI.

The notation used throughout the paper is fairly standard. The superscript " $T$ " stands for matrix transposition, $\mathbb{R}^{n}$ denotes the $n$-dimensional Euclidean space, 0 represents the zero matrix with appropriate dimensions, the notation $P>0$ $(\geq 0)$ means that $P$ is real symmetric and positive definite (semidefinite), $l_{2}[0, \infty)$ is the space of square-integrable vector functions over $[0, \infty)$, and $\|\cdot\|_{2}$ stands for the usual $l_{2}[0, \infty)$ norm. In symmetric block matrices or complex matrix expressions, we use an asterisk $(*)$ to represent a term that is induced by symmetry and $\operatorname{diag}\{\ldots\}$ stands for a block-diagonal matrix. In addition, $E\{x\}$ and $E\{x \mid y\}$ will, respectively, mean expectation of $x$ and expectation of $x$ conditional on $y$. Matrices, if their dimensions are not explicitly stated, are assumed to be compatible for algebraic operations.

\section{PROBlem Formulation}

Consider the fault detection problem for T-S fuzzy systems with intermittent measurements. The physical plant is repre- sented by a T-S fuzzy model, and the signal transmissions existing between the plant and the fault detection filter are intermittent.

\section{A. Physical Plant}

The nonlinear discrete-time system whose faults are to be detected is represented by the following T-S fuzzy model.

Plant rule $i$ : IF $\theta_{1}(k)$ is $M_{i 1}$ and $\theta_{2}(k)$ is $M_{i 2}$ and $\cdots$ and $\theta_{p}(k)$ is $M_{i p}$, THEN

$$
\begin{aligned}
x_{k+1}= & \left(A_{i}+\Delta A_{i}(k)\right) x_{k}+\left(B_{i}+\Delta B_{i}(k)\right) u_{k} \\
& +E_{1 i} w_{k}+E_{2 i} f_{k} \\
y_{k}= & C_{i} x_{k}+D_{i} u_{k}+F_{1 i} w_{k}+F_{2 i} f_{k}, \\
& i=1, \ldots, r
\end{aligned}
$$

where $M_{i j}$ is the fuzzy set, $x_{k} \in \mathbb{R}^{n_{p}}$ is the state vector; $r$ is the number of IF-THEN rules, $\theta(k)=\left[\theta_{1}(k), \theta_{2}(k), \ldots\right.$, $\left.\theta_{p}(k)\right]$ is the premise variable vector, $u_{k} \in \mathbb{R}^{m}$ is the deterministic input vector, $w_{k} \in \mathbb{R}^{p}$ is the exogenous disturbance input that belongs to $l_{2}[0, \infty)$, and $f_{k} \in \mathbb{R}^{q}$ is the fault vector that is also deterministic. Without the loss of generality, we assume that the $l_{2}$ norms of $u_{k}$ and $f_{k}$ exist and are bounded. $A_{i}, B_{i}, E_{1 i}, E_{2 i}, C_{i}, D_{i}, F_{1 i}$, and $F_{2 i}$ are known constant matrices with appropriate dimensions, $\Delta A_{i}(k)$ and $\Delta B_{i}(k)$ denote the uncertainties in the model and are of the form

$$
\begin{aligned}
\Delta A_{i}(k)=N_{i} Z(k) Q_{a i} \quad \Delta B_{i}(k)=N_{i} Z(k) Q_{b i}, & \\
i & =1, \ldots, r
\end{aligned}
$$

where $N_{i} \in \mathbb{R}^{n_{p} \times n_{z}}, Q_{a i} \in \mathbb{R}^{n_{z} \times n_{p}}$, and $Q_{b i} \in \mathbb{R}^{n_{z} \times m}$ are known constant matrices, and $Z(k) \in \mathbb{R}^{n_{z} \times n_{z}}$ is an unknown time-varying matrix with Lebesgue measurable elements bounded by

$$
Z^{T}(k) Z(k) \leq I
$$

Given a pair of $\left(x_{k}, u_{k}\right)$, the overall fuzzy system is inferred as

$$
\begin{gathered}
x_{k+1}=\sum_{i=1}^{r} h_{i}(\theta(k))\left[\left(A_{i}+\Delta A_{i}(k)\right) x_{k}+\left(B_{i}+\Delta B_{i}(k)\right) u_{k}\right. \\
\left.+E_{1 i} w_{k}+E_{2 i} f_{k}\right] \\
y_{k}=\sum_{i=1}^{r} h_{i}(\theta(k))\left[C_{i} x_{k}+D_{i} u_{k}+F_{1 i} w_{k}+F_{2 i} f_{k}\right]
\end{gathered}
$$

where $h_{i}(\theta(k))=\omega_{i}(\theta(k)) / \sum_{i=1}^{r} \omega_{i}(\theta(k))$ and $\omega_{i}(\theta(k))=$ $\prod_{j=1}^{p} M_{i j}\left(\theta_{j}(k)\right)$, with $M_{i j}\left(\theta_{j}(k)\right)$ representing the grade of membership of $\theta_{j}(k)$ in $M_{i j}$. Then, it can be seen that

$$
\omega_{i}\left(\theta_{k}\right) \geq 0, \quad i=1,2, \ldots, r, \quad \sum_{i=1}^{r} \omega_{i}\left(\theta_{k}\right)>0
$$

for all $k$.

\section{B. Fault Detection Filter}

One key step of fault detection is the generation of a residual signal, which must be sensitive to faults. This is often realized 
by utilizing fault detection observers [25], [26], [33] or filters [10], [14], [20], [33]. Since disturbances often inevitably appear in many systems, the residual signal must also be capable of distinguishing faults from exogenous signals. $H_{\infty}$ filter can not only describe the estimated signal accurately but also suppress the disturbance effectively. Thus, for the physical plant with disturbance in (1), we adopt the following fuzzy fault detection filter form, whose role is to generate residual signal based on the input $y_{f k}$.

Filter Rule $i$ : IF $\theta_{1}(k)$ is $M_{i 1}$ and $\theta_{2}(k)$ is $M_{i 2}$ and $\cdots$ and $\theta_{p}(k)$ is $M_{i p}$, THEN

$$
\begin{aligned}
\hat{x}_{k+1} & =A_{f i} \hat{x}_{k}+B_{f i} y_{f k} \\
r_{k} & =C_{f i} \hat{x}_{k}+D_{f i} y_{f k} \\
i & =1, \ldots, r .
\end{aligned}
$$

Here, $\hat{x}_{k} \in \mathbb{R}^{n_{f}}$ and $r_{k} \in R^{q}$, and $A_{f i}, B_{f i}, C_{f i}$, and $D_{f i}$ are to be determined. Thus, the filter can be represented by the following form:

$$
\begin{aligned}
\hat{x}_{k+1} & =\sum_{i=1}^{r} h_{i}(\theta(k))\left(A_{f i} \hat{x}_{k}+B_{f i} y_{f k}\right) \\
r_{k} & =\sum_{i=1}^{r} h_{i}(\theta(k))\left(C_{f i} \hat{x}_{k}+D_{f i} y_{f k}\right) .
\end{aligned}
$$

\section{Communication Links}

In this paper, we assume that a communication medium exists between the physical plant and the fault detection filter, and the data packet dropout phenomenon happens intermittently. Therefore, the measurement of the plant is no longer equivalent to the input of the filter (i.e., $y_{k} \neq y_{f k}$ ). A stochastic process is utilized to model the data loss phenomenon, i.e.

$$
y_{f k}=\alpha_{k} y_{k}
$$

where $\alpha_{k}$ is a Bernoulli process. When the link fails (i.e., data are lost), $\alpha_{k}=0$, and when the transmission is perfect, $\alpha_{k}=1$. A natural assumption on $\alpha_{k}$ can be made as

$$
\operatorname{Prob}\left\{\alpha_{k}=1\right\}=\mathbb{E}\left\{\alpha_{k}\right\}=\bar{\alpha} \quad \operatorname{Prob}\left\{\alpha_{k}=0\right\}=1-\bar{\alpha}
$$

where $\bar{\alpha}$ is assumed to be known. Based on this, we have

$$
\begin{aligned}
\hat{x}_{k+1} & =\sum_{i=1}^{r} h_{i}(\theta(k))\left(A_{f i} \hat{x}_{k}+\alpha_{k} B_{f i} y_{k}\right) \\
r_{k} & =\sum_{i=1}^{r} h_{i}(\theta(k))\left(C_{f i} \hat{x}_{k}+\alpha_{k} D_{f i} y_{k}\right) .
\end{aligned}
$$

Remark 1: The description of imperfect communication links existing between the plant and the fault detection filter follows that in the previous literature [28], [29]. The process of missing data considered is assumed to satisfy the Bernoulli distributed process. The probability distribution of the process is estimated based on experimental measurements of data transmitting from output of the plant to the input of the fault detection filter. This can be achieved by sending a sequence of indexed data through the communication medium and measuring the data dropout characteristics. The inferred statistics of the Bernoulli process will then be used for designing the fault detection filter.

\section{Fault Weighting System}

In fault detection, a reference residual model is usually needed to describe the desired behavior of the residual vector $r_{k}$. In this paper, the reference model is chosen as $\bar{f}(z)=W(z) f(z)$ [33], where $W(z)$ is given a priori. The choice of $W_{z}$ is to impose frequency weighting on the spectrum of the fault signal for detection. Here, we choose a stable matrix $W(z)$ to weight the fault signal $f_{k}$ [33], whose state-space realization is

$$
\begin{aligned}
\bar{x}_{k+1} & =A_{W} \bar{x}_{k}+B_{W} f_{k} \\
\bar{f}_{k} & =C_{W} \bar{x}_{k}+D_{W} f_{k}
\end{aligned}
$$

where $\bar{x}_{k} \in \mathbb{R}^{n_{W}}$ and $A_{W}, B_{W}, C_{W}$, and $D_{W}$ are priorly chosen.

\section{E. Residual Evaluation}

The residual evaluation function is to evaluate the generated residual. After the residual signal being constructed, a residual evaluation value will be computed through a prescribed evaluation function, and it will be compared with a predefined threshold. When the evaluation value is larger than the threshold, an alarm of fault is generated. Here, we consider the following evaluation function:

$$
\|r\|_{T} \triangleq \frac{1}{T} \sqrt{\sum_{k=t_{1}}^{t_{2}} r_{k}^{T} r_{k}} \quad T=t_{2}-t_{1}+1 .
$$

Choose a threshold $J_{\mathrm{th}}>0$, and for the detailed discussion of the threshold $J_{\mathrm{th}}$, readers are referred to [1] and [5]. The residual evaluation function value and the threshold satisfy the following relationship:

$$
\left\{\begin{array}{lll}
\|r\|_{T}>J_{\text {th }} & \Longrightarrow & \text { with faults } \quad \Longrightarrow \quad \text { alarm } \\
\|r\|_{T} \leq J_{\text {th }} & \Longrightarrow \quad \text { no faults. }
\end{array}\right.
$$

\section{F. Residual System}

From (4), (5), and (9), the residual system can be obtained as

$$
\begin{aligned}
\xi_{k+1}= & \sum_{i=1}^{r} \sum_{j=1}^{r} h_{i}(\theta(k)) h_{j}(\theta(k)) \\
& \times\left[\tilde{A}_{i j} \xi_{k}+\tilde{B}_{i j} \vartheta_{k}+\tilde{\alpha}_{k} \bar{A}_{1 i j} \xi_{k}+\tilde{\alpha}_{k} \bar{B}_{1 i j} \vartheta_{k}\right] \\
e_{k}= & \sum_{i=1}^{r} \sum_{j=1}^{r} h_{i}(\theta(k)) h_{j}(\theta(k)) \\
& \times\left[\bar{C}_{i j} \xi_{k}+\bar{D}_{i j} \vartheta_{k}+\tilde{\alpha}_{k} \bar{C}_{1 i j} \xi_{k}+\tilde{\alpha}_{k} \bar{D}_{1 i j} \vartheta_{k}\right]
\end{aligned}
$$

where

$$
\begin{aligned}
& \xi_{k}=\left[\begin{array}{lll}
\bar{x}_{k}^{T} & x_{k}^{T} & \hat{x}_{k}^{T}
\end{array}\right]^{T} \quad \vartheta_{k}=\left[\begin{array}{lll}
u_{k}^{T} & w_{k}^{T} & f_{k}^{T}
\end{array}\right]^{T} \\
& e_{k}=r_{k}-\bar{f}_{k}, \tilde{A}_{i j}=\left[\begin{array}{ccc}
A_{W} & 0 & 0 \\
0 & A_{i}+\Delta A_{i}(k) & 0 \\
0 & \bar{\alpha} B_{f i} C_{j} & A_{f i}
\end{array}\right]
\end{aligned}
$$




$$
\begin{aligned}
\tilde{B}_{i j}= & {\left[\begin{array}{ccc}
0 & 0 & B_{W} \\
B_{i}+\Delta B_{i}(k) & E_{1 i} & E_{2 i} \\
\bar{\alpha} B_{f i} D_{j} & \bar{\alpha} B_{f i} F_{1 j} & \bar{\alpha} B_{f i} F_{2 j}
\end{array}\right] } \\
\bar{A}_{1 i j}= & {\left[\begin{array}{ccc}
0 & 0 & 0 \\
0 & 0 & 0 \\
0 & B_{f i} C_{j} & 0
\end{array}\right] } \\
\bar{B}_{1 i j}= & {\left[\begin{array}{ccc}
0 & 0 & 0 \\
0 & 0 & 0 \\
B_{f i} D_{j} & B_{f i} F_{1 j} & B_{f i} F_{2 j}
\end{array}\right] } \\
\bar{C}_{i j}= & {\left[\begin{array}{lll}
-C_{W} & \bar{\alpha} D_{f i} C_{j} & C_{f i}
\end{array}\right] } \\
\bar{D}_{i j}= & {\left[\begin{array}{lll}
\bar{\alpha} D_{f i} D_{j} & \bar{\alpha} D_{f i} F_{1 j} & \bar{\alpha} D_{f i} F_{2 j}-D_{W}
\end{array}\right] } \\
\bar{C}_{1 i j}= & {\left[\begin{array}{lll}
0 & D_{f i} C_{j} & 0
\end{array}\right] } \\
\bar{D}_{1 i j}= & {\left[\begin{array}{lll}
D_{f i} D_{j} & D_{f i} F_{1 j} & D_{f i} F_{2 j}
\end{array}\right] }
\end{aligned}
$$

$\tilde{\alpha}_{k}=\alpha_{k}-\bar{\alpha}, E\left\{\tilde{\alpha}_{k}\right\}=0$ and $E\left\{\tilde{\alpha}_{k} \tilde{\alpha}_{k}\right\}=\bar{\alpha}(1-\bar{\alpha})$.

The residual system presents the difference between the generated residual and the idealized reference residual signal. By minimizing the $H_{\infty}$ norm of the difference, the effect of the disturbance can be minimized and the sensitivity of the residual to fault can be maximized [1], [18], [33]. Therefore, design of the fault detection filter can be converted as an $H_{\infty}$ model matching problem [33].

Then, the problem to be addressed in this paper is expressed as follows.

Problem: Fuzzy fault detection with intermittent measurements (FFDIMs): Consider the fuzzy system in (4), and suppose that the intermittent transmission parameter $\bar{\alpha}$ is known. Given a scalar $\gamma>0$, design a fuzzy fault detection filter in the form of (5) such that:

1) the residual system in (10) is stochastically stable; and

2) under zero initial conditions, the residual error $e_{k}$ satisfies

$$
\|e\|_{E} \leq \gamma\|\vartheta\|_{2}
$$

where $\|e\|_{E} \triangleq E\left\{\sqrt{\sum_{k=0}^{\infty} e_{k}^{T} e_{k}}\right\}$ and $\|\vartheta\|_{2} \triangleq\left(\sum_{k=0}^{\infty} \vartheta_{k}^{T}\right.$ $\left.\vartheta_{k}\right)^{\frac{1}{2}}$. It is noted that the $l_{2}$ norm of $\vartheta_{k}$ exists and is bounded since its constituent variables are all $l_{2}$ norm bounded.

Before proceeding further, we first introduce the following definition.

Definition 1: The residual system in (10) is said to be stochastically stable in the mean square $\left(\vartheta_{k} \equiv 0\right)$ if there exists a finite $V>0$ independent of $\xi_{0}$, such that for any initial condition $\xi_{0} E\left\{\sum_{k=0}^{\infty} \xi_{k}^{T} \xi_{k} \mid \xi_{0}\right\}<\xi_{0}^{T} V \xi_{0}$.

Remark 2: Different statements of stochastic stability definitions are presented in [22], which serve for different systems. They have the same criterion that the expectation values of all the solutions of the system must be energy-bounded when the operation time is infinite. The solutions are generally dependent on initial conditions or other elements, and thus, the condition expectation is often used. The definition proposed here also obeys the criterion.

\section{FuZzy Fault Detection FiLter Design FOR NOMINAL SYSTEMS}

In this section, the FFDIM problem is solved for the nominal fuzzy system in (12). The fault detection analysis problem is first solved, and then, based on that, a full-rank fault detection filter is designed (i.e., $n_{f}=n_{p}+n_{W}$ ). The fuzzy basis-dependent technique is utilized, which potentially reduces the conservatism of the obtained results.

The nominal system of (4) takes the following form:

$$
\begin{aligned}
x_{k+1} & =\sum_{i=1}^{r} h_{i}(\theta(k))\left[A_{i} x_{k}+B_{i} u_{k}+E_{1 i} w_{k}+E_{2 i} f_{k}\right] \\
y_{k} & =\sum_{i=1}^{r} h_{i}(\theta(k))\left[C_{i} x_{k}+D_{i} u_{k}+F_{1 i} w_{k}+F_{2 i} f_{k}\right]
\end{aligned}
$$

and the nominal residual fuzzy system is given by

$$
\begin{aligned}
\xi_{k+1}= & \sum_{i=1}^{r} \sum_{j=1}^{r} h_{i}(\theta(k)) h_{j}(\theta(k)) \\
& \times\left[\bar{A}_{i j} \xi_{k}+\bar{B}_{i j} \vartheta_{k}+\tilde{\alpha}_{k} \bar{A}_{1 i j} \xi_{k}+\tilde{\alpha}_{k} \bar{B}_{1 i j} \vartheta_{k}\right] \\
e_{k}= & \sum_{i=1}^{r} \sum_{j=1}^{r} h_{i}(\theta(k)) h_{j}(\theta(k)) \\
& \times\left[\bar{C}_{i j} \xi_{k}+\bar{D}_{i j} \vartheta_{k}+\tilde{\alpha}_{k} \bar{C}_{1 i j} \xi_{k}+\tilde{\alpha}_{k} \bar{D}_{1 i j} \vartheta_{k}\right]
\end{aligned}
$$

where

$$
\begin{aligned}
\bar{A}_{i j} & =\left[\begin{array}{ccc}
A_{W} & 0 & 0 \\
0 & A_{i} & 0 \\
0 & \bar{\alpha} B_{f i} C_{j} & A_{f i}
\end{array}\right] \\
\bar{B}_{i j} & =\left[\begin{array}{ccc}
0 & 0 & B_{W} \\
B_{i} & E_{1 i} & E_{2 i} \\
\bar{\alpha} B_{f i} D_{j} & \bar{\alpha} B_{f i} F_{1 j} & \bar{\alpha} B_{f i} F_{2 j}
\end{array}\right] .
\end{aligned}
$$

\section{A. Fault Detection Analysis}

In this section, we assume that the fault detection filter matrices in (5) are known, and the conditions are investigated under which the residual system is stochastically stable and guarantees the performance defined in (11). The following theorem tells us that the performance of the residual system can be guaranteed if there exist some matrices satisfying certain LMIs.

Theorem 1: Consider the fuzzy system in (12) and suppose the fault detection filter matrices $A_{f i}, B_{f i}, C_{f i}$, and $D_{f i}$ in (6) are known. The residual system in (13) is stochastically stable with a guaranteed performance $\gamma$ if there exist $n$-dimensional matrices $P_{l}>0$ for $l=1, \ldots, r$, where $n=n_{f}+n_{W}+n_{p}$, and an 
$n$-dimensional matrix $G$ satisfying the following inequality:

$$
\left[\begin{array}{cccccc}
\Pi_{l} & 0 & 0 & 0 & G^{T} \bar{A}_{i j} & G^{T} \bar{B}_{i j} \\
* & \Pi_{l} & 0 & 0 & f G^{T} \bar{A}_{1 i j} & f G^{T} \bar{B}_{1 i j} \\
* & * & -I & 0 & \bar{C}_{i j} & \bar{D}_{i j} \\
* & * & * & -I & f \bar{C}_{1 i j} & f \bar{D}_{1 i j} \\
* & * & * & * & -P_{i} & 0 \\
* & * & * & * & * & -\gamma^{2} I
\end{array}\right]<0
$$

where $f=\sqrt{\bar{\alpha}(1-\bar{\alpha})}$ and $\Pi_{l}=P_{l}-G-G^{T}$.

Proof: Suppose there exist real symmetric positive definite matrices $P_{l}$ for $l=1, \ldots, r$ and a nonsingular matrix $G$ satisfying (14). Noting the inequality $\left(P_{l}-G\right)^{T} P_{l}^{-1}\left(P_{l}-G\right) \geq 0$ implies $P_{l}-G-G^{T} \geq-G^{T} P_{l}^{-1} G$, which together with (14) yields

$$
\left[\begin{array}{cccccc}
\tilde{\Pi}_{i} & 0 & 0 & 0 & G^{T} \bar{A}_{i j} & G^{T} \bar{B}_{i j} \\
* & \tilde{\Pi}_{i} & 0 & 0 & f G^{T} \bar{A}_{1 i j} & f G^{T} \bar{B}_{1 i j} \\
* & * & -I & 0 & \bar{C}_{i j} & \bar{D}_{i j} \\
* & * & * & -I & f \bar{C}_{1 i j} & f \bar{D}_{1 i j} \\
* & * & * & * & -P_{i} & 0 \\
* & * & * & * & * & -\gamma^{2} I
\end{array}\right]<0
$$

where $\tilde{\Pi}_{l}=-G^{T} P_{l}^{-1} G$.

Pre- and postmultiplying $\operatorname{diag}\left\{G^{-T}, G^{-T}, I, I, I, I\right\}$ and $\operatorname{diag}\left\{G^{-1}, G^{-1}, I, I, I, I\right\}$ to (15) and by Schur complement, we have

$$
\begin{array}{r}
{\left[\begin{array}{cc}
\bar{A}_{i j}^{T} & f \bar{A}_{1 i j}^{T} \\
\bar{B}_{i j}^{T} & f \bar{B}_{1 i j}^{T}
\end{array}\right]\left[\begin{array}{cc}
P_{l} & 0 \\
0 & P_{l}
\end{array}\right]\left[\begin{array}{cc}
\bar{A}_{i j} & \bar{B}_{i j} \\
f \bar{A}_{1 i j} & f \bar{B}_{1 i j}
\end{array}\right]+\left[\begin{array}{cc}
\bar{C}_{i j}^{T} & f \bar{C}_{1 i j}^{T} \\
\bar{D}_{i j}^{T} & f \bar{D}_{1 i j}^{T}
\end{array}\right]} \\
\times\left[\begin{array}{cc}
\bar{C}_{i j} & \bar{D}_{i j} \\
f \bar{C}_{1 i j} & f \bar{D}_{1 i j}
\end{array}\right]-\left[\begin{array}{cc}
P_{i} & 0 \\
* & \gamma^{2} I
\end{array}\right]<0 .
\end{array}
$$

Now, we first prove the stochastic stability of the residual system in (13). Define an index as

$J=E\left\{\xi_{k+1}^{T} \sum_{l=1}^{r} h_{l}(\theta(k+1)) P_{l} \xi_{k+1} \mid \xi_{k}\right\}-\xi_{k}^{T} \sum_{i=1}^{r} h_{i}(\theta(k)) P_{i} \xi_{k}$.

When $\vartheta_{k} \equiv 0$, along the nominal system in (13), $J$ gives

$$
\begin{aligned}
J=E\{ & \sum_{l=1}^{r} h_{l}(\theta(k+1)) \sum_{i=1}^{r} \sum_{j=1}^{r} \sum_{s=1}^{r} \sum_{t=1}^{r} h_{i}(\theta(k)) h_{j}(\theta(k)) \\
& \times h_{s}(\theta(k)) h_{t}(\theta(k))\left[\bar{A}_{i j} \xi_{k}+\tilde{\alpha}_{k} \bar{A}_{1 i j} \xi_{k}\right]^{T} P_{l} \\
& \left.\times\left[\bar{A}_{s t} \xi_{k}+\tilde{\alpha}_{k} \bar{A}_{1 s t} \xi_{k}\right] \mid \xi_{k}\right\}-\xi_{k}^{T} \sum_{i=1}^{r} h_{i}(\theta(k)) P_{i} \xi_{k} \\
\leq \xi_{k}^{T} & \sum_{l=1}^{r} h_{l}(\theta(k+1)) \sum_{i=1}^{r} \sum_{j=1}^{r} h_{i}(\theta(k)) h_{j}(\theta(k)) \\
& \times\left\{\bar{A}_{i j}^{T} P_{l} \bar{A}_{i j}+\bar{\alpha}(1-\bar{\alpha}) \bar{A}_{1 i j}^{T} P_{l} \bar{A}_{1 i j}-P_{i}\right\} \xi_{k} . \quad \text { (18) }
\end{aligned}
$$

Define

$$
\begin{aligned}
\bar{\Psi}_{i j l} & =\bar{A}_{i j}^{T} P_{l} \bar{A}_{i j}+\bar{\alpha}(1-\bar{\alpha}) \bar{A}_{1 i j}^{T} P_{l} \bar{A}_{1 i j}-P_{i} \\
\Psi(k) & =\sum_{l=1}^{r} h_{l}(\theta(k+1)) \sum_{i=1}^{r} \sum_{j=1}^{r} h_{i}(\theta(k)) h_{j}(\theta(k)) \bar{\Psi}_{i j l} .
\end{aligned}
$$

Then, the derivative process in (18) concludes $J \leq \xi_{k}^{T} \Psi(k) \xi_{k}$, i.e.

$$
\begin{aligned}
E\left\{\xi_{k+1}^{T} \sum_{l=1}^{r} h_{l}(\right. & \left.(k+1)) P_{l} \xi_{k+1} \mid \xi_{k}\right\} \\
& -\xi_{k}^{T} \sum_{i=1}^{r} h_{i}(\theta(k)) P_{i} \xi_{k} \leq \xi_{k}^{T} \Psi(k) \xi_{k} .
\end{aligned}
$$

Taking mathematical expectation and summing up the terms on both sides of (19) for $k=0, \ldots, \beta$, for any $\beta>1$, we have

$$
\begin{gathered}
E\left\{\xi_{\beta+1}^{T} \sum_{l=1}^{r} h_{l}(\theta(\beta+1)) P_{l} \xi_{\beta+1}\right\}-\xi_{0}^{T} \sum_{i=1}^{r} h_{i}(\theta(0)) P_{i} \xi_{0} \\
\leq E\left\{\sum_{k=0}^{\beta} \sum_{l=1}^{r} h_{l}(\theta(\beta+1)) \sum_{i=1}^{r} \sum_{j=1}^{r} h_{i}(\theta(k)) h_{j}(\theta(k)) \xi_{k}^{T} \bar{\Psi}_{i j l} \xi_{k}\right\} \\
\leq E\left\{\sum_{k=0}^{\beta} \sum_{l=1}^{r} h_{l}(\theta(\beta+1)) \sum_{i=1}^{r} \sum_{j=1}^{r} h_{i}(\theta(k)) h_{j}(\theta(k))\right. \\
\left.\times\left(\lambda_{\max }\left(\bar{\Psi}_{i j l}\right) \xi_{k}^{T} \xi_{k}\right)\right\}
\end{gathered}
$$

$=E\left\{\sum_{k=0}^{\beta} \max _{i, j, l=1, \ldots, r}\left(\lambda_{\max }\left(\bar{\Psi}_{i j l}\right)\right) \xi_{k}^{T} \xi_{k}\right\}$.

From the aforementioned inequalities, it is not difficult to conclude that, for $i, j, l=1, \ldots, r$ and $\beta$, the following inequality is true:

$$
\begin{aligned}
E & \left\{\xi_{\beta+1}^{T} P_{l} \xi_{\beta+1}\right\}-\xi_{0}^{T} P_{i} \xi_{0} \\
& \leq \max _{i, j, l=1, \ldots, r}\left(\lambda_{\max }\left(\bar{\Psi}_{i j l}\right)\right) E\left\{\sum_{k=0}^{\beta} \xi_{k}^{T} \xi_{k}\right\} .
\end{aligned}
$$

When $\beta \rightarrow \infty$, we obtain

$$
\begin{aligned}
E & \left\{\xi_{\infty}^{T} P_{l} \xi_{\infty}\right\}-\xi_{0}^{T} P_{i} \xi_{0} \\
& \leq \max _{i, j, l=1, \ldots, r}\left(\lambda_{\max }\left(\bar{\Psi}_{i j l}\right)\right) E\left\{\sum_{k=0}^{\infty} \xi_{k}^{T} \xi_{k}\right\} .
\end{aligned}
$$

Considering nonzero initial condition and $E\left\{\xi_{\infty}^{T} P_{l} \xi_{\infty}\right\} \geq 0$, we have

$$
\begin{aligned}
& E\left\{\sum_{k=0}^{\infty} \xi_{k}^{T} \xi_{k} \mid \xi_{0}\right\} \leq\left(-\max _{i, j, l=1, \ldots, r}\left(\lambda_{\max }\left(\bar{\Psi}_{i j l}\right)\right)\right)^{-1} \xi_{0}^{T} P_{i} \xi_{0} \\
& \quad \leq \xi_{0}^{T}\left(-\left(\max _{i, j, l=1, \ldots, r}\left(\lambda_{\max }\left(\bar{\Psi}_{i j l}\right)\right)\right)^{-1} \max _{i=1, \ldots, r}\left(\lambda_{\max }\left(P_{i}\right)\right)\right) \xi_{0} \\
& \quad=\sigma \xi_{0}^{T} \xi_{0}
\end{aligned}
$$


where

$$
\sigma \triangleq-\left(\max _{i, j, l=1, \ldots, r}\left(\lambda_{\max }\left(\bar{\Psi}_{i j l}\right)\right)\right)^{-1} \max _{i=1, \ldots, r}\left(\lambda_{\max }\left(P_{i}\right)\right)
$$

and $\xi_{0}$ is the initial condition. From $(1,1)$ block in the left side of (16), $\bar{\Psi}_{i j l}<0$ is obtained, and thus, $\sigma>0$. According to Definition 1 , the residual system is stochastically stable in the mean square.

Next, we prove that the performance defined in (11) is guaranteed. To this end, assume zero initial condition and $\vartheta_{k} \neq 0$. An index is introduced as

$$
\begin{aligned}
\bar{J}= & E\left\{\xi_{k+1}^{T} \sum_{l=1}^{r} h_{l}(\theta(k+1)) P_{l} \xi_{k+1} \mid \xi_{k}\right\} \\
& -\xi_{k}^{T} \sum_{i=1}^{r} h_{i}(\theta(k)) P_{i} \xi_{k}+E\left\{e_{k}^{T} e_{k} \mid \xi_{k}\right\}-\gamma^{2} \vartheta_{k}^{T} \vartheta_{k} .
\end{aligned}
$$

Along the nominal system in (13), we have

$$
\begin{aligned}
& E\left\{\xi_{k+1}^{T} \sum_{l=1}^{r} h_{l}(\theta(k+1)) P_{l} \xi_{k+1} \mid \xi_{k}\right\} \\
& =E\left\{\sum_{l=1}^{r} h_{l}(\theta(k+1)) \sum_{i=1}^{r} \sum_{j=1}^{r} \sum_{s=1}^{r} \sum_{t=1}^{r} h_{i}(\theta(k)) h_{j}(\theta(k))\right. \\
& \times h_{s}(\theta(k)) h_{t}(\theta(k)) \eta_{k}^{T}\left(\left[\begin{array}{c}
\bar{A}_{i j}^{T} \\
\bar{B}_{i j}^{T}
\end{array}\right]+\tilde{\alpha}_{k}\left[\begin{array}{c}
\bar{A}_{1 i j}^{T} \\
\bar{B}_{1 i j}^{T}
\end{array}\right]\right) \\
& \left.\times P_{l}\left(\left[\begin{array}{ll}
\bar{A}_{s t} & \bar{B}_{s t}
\end{array}\right]+\tilde{\alpha}_{k}\left[\begin{array}{ll}
\bar{A}_{1 s t} & \bar{B}_{1 s t}
\end{array}\right]\right) \eta_{k} \mid \xi_{k}\right\} \\
& \leq \sum_{l=1}^{r} h_{l}(\theta(k+1)) \sum_{i=1}^{r} \sum_{j=1}^{r} h_{i}(\theta(k)) h_{j}(\theta(k)) \eta_{k}^{T} \\
& \times\left\{\left[\begin{array}{c}
\bar{A}_{i j}^{T} \\
\bar{B}_{i j}^{T}
\end{array}\right] P_{l}\left[\begin{array}{ll}
\bar{A}_{i j} & \bar{B}_{i j}
\end{array}\right]+f^{2}\left[\begin{array}{c}
\bar{A}_{1 i j}^{T} \\
\bar{B}_{1 i j}^{T}
\end{array}\right] P_{l}\left[\begin{array}{ll}
\bar{A}_{1 i j} & \bar{B}_{1 i j}
\end{array}\right]\right\} \eta_{k} \\
& E\left\{e_{k}^{T} e_{k} \mid \xi_{k}\right\} \\
& =E\left\{\sum_{i=1}^{r} \sum_{j=1}^{r} \sum_{s=1}^{r} \sum_{t=1}^{r} h_{i}(\theta(k)) h_{j}(\theta(k))\right. \\
& \times h_{s}(\theta(k)) h_{t}(\theta(k)) \eta_{k}^{T}\left(\left[\begin{array}{c}
\bar{C}_{i j}^{T} \\
\bar{D}_{i j}^{T}
\end{array}\right]+\tilde{\alpha}_{k}\left[\begin{array}{c}
\bar{C}_{1 i j}^{T} \\
\bar{D}_{1 i j}^{T}
\end{array}\right]\right) \\
& \left.\times\left(\left[\begin{array}{cc}
\bar{C}_{s t} & \bar{D}_{s t}
\end{array}\right]+\tilde{\alpha}_{k}\left[\begin{array}{ll}
\bar{C}_{1 s t} & \bar{D}_{1 s t}
\end{array}\right]\right) \eta_{k} \mid \xi_{k}\right\} \\
& \leq \eta_{k}^{T} \sum_{i=1}^{r} \sum_{j=1}^{r} h_{i}(\theta(k)) h_{j}(\theta(k)) \\
& \times\left(\left[\begin{array}{c}
\bar{C}_{i j}^{T} \\
\bar{D}_{i j}^{T}
\end{array}\right]\left[\begin{array}{ll}
\bar{C}_{i j} & \bar{D}_{i j}
\end{array}\right]+f^{2}\left[\begin{array}{c}
\bar{C}_{1 i j}^{T} \\
\bar{D}_{1 i j}^{T}
\end{array}\right]\left[\begin{array}{ll}
\bar{C}_{1 i j} & \bar{D}_{1 i j}
\end{array}\right]\right) \eta_{k}
\end{aligned}
$$

where $\eta_{k}=\left[\begin{array}{ll}\xi_{k}^{T} & \vartheta_{k}^{T}\end{array}\right]^{T}$. By substituting (21) into (20), the following holds:

$$
\begin{aligned}
& \bar{J} \leq \sum_{l=1}^{r} h_{l}(\theta(k+1)) \sum_{i=1}^{r} \sum_{j=1}^{r} h_{i}(\theta(k)) h_{j}(\theta(k)) \\
& \times \eta_{k}^{T}\left\{\left[\begin{array}{c}
\bar{A}_{i j}^{T} \\
\bar{B}_{i j}^{T}
\end{array}\right] P_{l}\left[\begin{array}{ll}
\bar{A}_{i j} & \bar{B}_{i j}
\end{array}\right]+f^{2}\left[\begin{array}{c}
\bar{A}_{1 i j}^{T} \\
\bar{B}_{1 i j}^{T}
\end{array}\right] P_{l}\right. \\
& \times\left[\begin{array}{ll}
\bar{A}_{1 i j} & \bar{B}_{1 i j}
\end{array}\right]+\left[\begin{array}{c}
\bar{C}_{i j}^{T} \\
\bar{D}_{i j}^{T}
\end{array}\right]\left[\begin{array}{ll}
\bar{C}_{i j} & \bar{D}_{i j}
\end{array}\right] \\
& \left.+f^{2}\left[\begin{array}{c}
\bar{C}_{1 i j}^{T} \\
\bar{D}_{1 i j}^{T}
\end{array}\right]\left[\begin{array}{ll}
\bar{C}_{1 i j} & \bar{D}_{1 i j}
\end{array}\right]\right\} \eta_{k}-\eta_{k}^{T}\left[\begin{array}{cc}
P_{i} & 0 \\
0 & \gamma^{2} I
\end{array}\right] \eta_{k}
\end{aligned}
$$

which leads to $\bar{J} \leq 0$ by consideration of (16), i.e.

$$
\begin{aligned}
& E\left\{\xi_{k+1}^{T} \sum_{l=1}^{r} h_{l}(\theta(k+1)) P_{l} \xi_{k+1} \mid \xi_{k}\right\}-\xi_{k}^{T} \sum_{i=1}^{r} h_{i}(\theta(k)) P_{i} \xi_{k} \\
& +E\left\{e_{k}^{T} e_{k} \mid \xi_{k}\right\}-\gamma^{2} \vartheta_{k}^{T} \vartheta_{k} \leq 0 .
\end{aligned}
$$

Taking mathematical expectation on both sides of (22), we obtain

$$
\begin{aligned}
E & \left\{\xi_{k+1}^{T} \sum_{l=1}^{r} h_{l}(\theta(k+1)) P_{l} \xi_{k+1}\right\} \\
& -E\left\{\xi_{k}^{T} \sum_{i=1}^{r} h_{i}(\theta(k)) P_{i} \xi_{k}\right\}+E\left\{e_{k}^{T} e_{k}\right\}-\gamma^{2} \vartheta_{k}^{T} \vartheta_{k} \leq 0 .
\end{aligned}
$$

For $k=0,1,2, \ldots$, summing up both sides of the aforementioned inequality, considering zero initial condition and $E\left\{\xi_{\infty}^{T} \sum_{i=1}^{r} h_{i}(\theta(\infty)) P_{i} \xi_{\infty}\right\}>0$, we have

$$
E\left\{\sum_{k=0}^{\infty} e_{k}^{T} e_{k}\right\}-\gamma^{2} \sum_{k=0}^{\infty} \vartheta_{k}^{T} \vartheta_{k} \leq 0
$$

which is equivalent to the inequality in (11), and thus, the proof is completed.

Remark 3: Results of fault detection for fuzzy systems provide feasible solutions to the problem of fault detection for nonlinear systems [17], which are useful in practice since most physical systems in the real world are nonlinear. Previous results are mostly concerned with the perfect communication links. Actually, in practice, the transmission is often imperfect between the plant and the filter, i.e., data packet dropout may occur intermittently, especially in systems based on the network communication links. In this paper, data missing is considered in the fuzzy model, which makes the obtained results more general and practical.

\section{B. Fault Detection Filter Design}

In this section, the fault detection filter design problem will be investigated based on Theorem 1, i.e., a method will be developed to determine the fault detection filter matrices in (5), such that the residual system in (13) is stochastically stable and the performance defined in (11) is guaranteed. 
Theorem 2: Consider the fuzzy system in (12). For a given positive constant $\gamma$, if there exist $n$-dimensional matrices

$$
\tilde{P}_{l}=\left[\begin{array}{cc}
\tilde{P}_{1 l} & \tilde{P}_{2 l} \\
\tilde{P}_{2 l}^{T} & \tilde{P}_{3 l}
\end{array}\right]>0,
$$

where $n=n_{f}+n_{W}+n_{p}$, matrices $\breve{A}_{f i}, \breve{B}_{f i}, \breve{C}_{f i}$, and $\breve{D}_{f i}$, for any $i, l=1, \ldots, r$, and $\left(n_{p}+n_{W}\right)$-dimensional matrices $U, X$, and $W$ satisfy the following inequality:

$$
\Phi_{i j l}=\left[\begin{array}{cccccc}
\Theta_{l} & 0 & 0 & 0 & \Theta_{15 i j} & \Theta_{16 i j} \\
* & \Theta_{l} & 0 & 0 & \Theta_{25 i j} & \Theta_{26 i j} \\
* & * & -I & 0 & \Theta_{35 i j} & \Theta_{36 i j} \\
* & * & * & -I & \Theta_{45 i j} & \Theta_{46 i j} \\
* & * & * & * & \Theta_{55 i} & 0 \\
* & * & * & * & * & -\gamma^{2} I
\end{array}\right]<0
$$

where

$$
\begin{aligned}
& \Theta_{l}=\tilde{P}_{l}-\Omega-\Omega^{T} \quad \Omega=\left[\begin{array}{cc}
U & X \\
W^{T} & W^{T}
\end{array}\right] \\
& \Theta_{15 i j}=\left[\begin{array}{ccc}
U^{T} \breve{A}_{i}+\bar{\alpha} \breve{B}_{f i} \breve{C}_{j} & \breve{A}_{f i} \\
X^{T} \breve{A}_{i}+\bar{\alpha} \breve{B}_{f i} \breve{C}_{j} & \breve{A}_{f i}
\end{array}\right] \quad \Theta_{25 i j}=\left[\begin{array}{ccc}
f \breve{B}_{f i} \breve{C}_{j} & 0 \\
f \breve{B}_{f i} \breve{C}_{j} & 0
\end{array}\right] \\
& \Theta_{45 i j}=\left[\begin{array}{lll}
f \breve{D}_{f i} \breve{C}_{j} & 0
\end{array}\right] \quad \Theta_{35 i j}=\left[\begin{array}{lll}
\bar{\alpha} \breve{D}_{f i} \breve{C}_{j}-C_{W} & \breve{C}_{f i}
\end{array}\right] \\
& \Theta_{55 i}=\left[\begin{array}{ll}
-\tilde{P}_{1 i} & -\tilde{P}_{2 i} \\
-\tilde{P}_{2 i}^{T} & -\tilde{P}_{3 i}
\end{array}\right] \quad \Theta_{16 i j}=\left[\begin{array}{l}
U^{T} \breve{B}_{1 i}+\bar{\alpha} \breve{B}_{f i} \breve{D}_{j} \\
X^{T} \breve{B}_{1 i}+\bar{\alpha} \breve{B}_{f i} \breve{D}_{j}
\end{array}\right] \\
& \Theta_{26 i j}=\left[\begin{array}{cc}
f \breve{B}_{f i} \breve{D}_{j} \\
f \breve{B}_{f i} \breve{D}_{j}
\end{array}\right] \quad \Theta_{36 i j}=\left[\bar{\alpha} \breve{D}_{f i} \breve{D}_{j}-\breve{D}_{W}\right] \\
& \Theta_{46 i j}=f \breve{D}_{f i} \breve{D}_{j} \quad \breve{A}_{i}=\left[\begin{array}{cc}
A_{W} & 0 \\
0 & A_{i}
\end{array}\right] \\
& \breve{B}_{1 i}=\left[\begin{array}{ccc}
0 & 0 & B_{W} \\
B_{i} & E_{1 i} & E_{2 i}
\end{array}\right] \quad \breve{D}_{f i}=D_{f i} \quad \breve{C}_{i}=\left[\begin{array}{ll}
0 & C_{i}
\end{array}\right] \\
& \breve{D}_{i}=\left[\begin{array}{lll}
D_{i} & F_{1 i} & F_{2 i}
\end{array}\right] \quad \breve{D}_{W}=\left[\begin{array}{lll}
0 & 0 & D_{W}
\end{array}\right] \text {. }
\end{aligned}
$$

then there exists a fuzzy fault detection filter in the form of (6), such that the residual system in (13) is stochastically stable with the performance $\gamma$ defined in (11). Moreover, if the aforementioned conditions are satisfied, the matrices for the fault detection filter in (6) are given by

$$
\left[\begin{array}{cc}
A_{f i} & B_{f i} \\
C_{f i} & D_{f i}
\end{array}\right]=\left[\begin{array}{cc}
G_{4}^{-T} & 0 \\
0 & I
\end{array}\right]\left[\begin{array}{ll}
\breve{A}_{f i} & \breve{B}_{f i} \\
\breve{C}_{f i} & \breve{D}_{f i}
\end{array}\right]\left[\begin{array}{cc}
V^{-1} & 0 \\
0 & I
\end{array}\right]
$$

with $G_{4}$ and $V$ being nonsingular matrices and satisfying $W=$ $G_{4}^{T} V$.

Proof: Suppose there exist real symmetric positive definite matrices $\tilde{P}_{l}$ for $l=1, \ldots r$, matrices $U, X$, and $W$ satisfy (23).
From (23), we know that

$$
\tilde{P}_{l}<\Omega+\Omega^{T}
$$

which implies $\Omega$ and $W$ are nonsingular. One can always find square and nonsingular matrices $G_{3}$ and $G_{4}$ such that $W=$ $G_{4}^{T} G_{3}^{-1} G_{4}$. Let

$G_{1}=U \quad X=G_{2}^{T} G_{3}^{-1} G_{4} \quad V=G_{3}^{-1} G_{4} \quad G=\left[\begin{array}{ll}G_{1} & G_{2} \\ G_{4} & G_{3}\end{array}\right]$

and define a transposition matrix

$$
T=\left[\begin{array}{cc}
I & 0 \\
0 & G_{3}^{-1} G_{4}
\end{array}\right] .
$$

Without loss of generality, we can assume

$$
\left[\begin{array}{ll}
\tilde{P}_{1 i} & \tilde{P}_{2 i} \\
\tilde{P}_{2 i}^{T} & \tilde{P}_{3 i}
\end{array}\right]=T^{T} P_{i} T .
$$

From (25) and (26), we know that

$$
\left[\begin{array}{cc}
\breve{A}_{f i} & \breve{B}_{f i} \\
\breve{C}_{f i} & \breve{D}_{f i}
\end{array}\right]=\left[\begin{array}{cc}
G_{4}^{T} & 0 \\
0 & I
\end{array}\right]\left[\begin{array}{cc}
A_{f i} & B_{f i} \\
C_{f i} & D_{f i}
\end{array}\right]\left[\begin{array}{cc}
G_{3}^{-1} G_{4} & 0 \\
0 & I
\end{array}\right] .
$$

Substituting (26)-(29) into (23), we have

$$
\begin{aligned}
& \Theta_{l}=T^{T}\left(P_{l}-G-G^{T}\right) T=\tilde{P}_{l}-\Omega-\Omega^{T} \\
& \Theta_{15 i j}=\left[\begin{array}{cc}
G_{1}^{T} \breve{A}_{i}+\bar{\alpha} G_{4}^{T} B_{f i} \breve{C}_{j} & G_{4}^{T} A_{f i} G_{3}^{-1} G_{4} \\
G_{4}^{T} G_{3}^{-T} G_{2} \breve{A}_{i}+\bar{\alpha} G_{4}^{T} B_{f i} \breve{C}_{j} & G_{4}^{T} A_{f i} G_{3}^{-1} G_{4}
\end{array}\right] \\
& \Theta_{25 i j}=\left[\begin{array}{ll}
f G_{4}^{T} B_{f i} \breve{C}_{j} & 0 \\
f G_{4}^{T} B_{f i} \breve{C}_{j} & 0
\end{array}\right] \quad \Theta_{45 i j}=\left[\begin{array}{ll}
f D_{f i} \breve{C}_{j} & 0
\end{array}\right] \\
& \Theta_{35 i j}=\left[\bar{\alpha} D_{f i} \breve{C}_{j}-C_{W} \quad C_{f i} G_{3}^{-1} G_{4}\right] \\
& \Theta_{55 i j}=T^{T}\left[\begin{array}{ll}
-P_{1 i} & -P_{2 i} \\
-P_{2 i}^{T} & -P_{3 i}
\end{array}\right] T \\
& \Theta_{16 i j}=\left[\begin{array}{c}
U^{T} \breve{B}_{1 i}+\bar{\alpha} G_{4}^{T} B_{f i} \breve{D}_{j} \\
G_{4}^{T} G_{3}^{-T} G_{2} \breve{B}_{1 i}+\bar{\alpha} G_{4}^{T} B_{f i} \breve{D}_{j}
\end{array}\right] \\
& \Theta_{26 i j}=\left[\begin{array}{l}
f G_{4}^{T} B_{f i} \breve{D}_{j} \\
f G_{4}^{T} B_{f i} \breve{D}_{j}
\end{array}\right] \quad \Theta_{36 i j}=\left[\bar{\alpha} D_{f i} \breve{D}_{j}-\breve{D}_{W}\right] \\
& \Theta_{46 i j}=f D_{f i} \breve{D}_{j} \text {. }
\end{aligned}
$$

Then, one can conclude that the inequality in (23) is equivalent to (30), as shown at the bottom of this page, which clearly guarantees the inequality in (14). Thus, the proof is completed.

Remark 4: Without loss of generality, we assume the number of rows in $C_{W}$ is $n_{C_{W}}$. Then, the two identity matrices in (3,3)

$$
\left[\begin{array}{cccccc}
T^{T} & 0 & 0 & 0 & 0 & 0 \\
0 & T^{T} & 0 & 0 & 0 & 0 \\
0 & 0 & I & 0 & 0 & 0 \\
0 & 0 & 0 & I & 0 & 0 \\
0 & 0 & 0 & 0 & T^{T} & 0 \\
0 & 0 & 0 & 0 & 0 & I
\end{array}\right]\left[\begin{array}{cccccc}
\Pi_{l} & 0 & 0 & 0 & G^{T} \bar{A}_{i j} & G^{T} \bar{B}_{i j} \\
* & \Pi_{l} & 0 & 0 & f G^{T} \bar{A}_{1 i j} & f G^{T} \bar{B}_{1 i j} \\
* & * & -I & 0 & \bar{C}_{i j} & \bar{D}_{i j} \\
* & * & * & -I & f \bar{C}_{1 i j} & f \bar{D}_{1 i j} \\
* & * & * & * & -P_{i} & 0 \\
* & * & * & * & * & -\gamma^{2} I
\end{array}\right]\left[\begin{array}{cccccc}
T & 0 & 0 & 0 & 0 & 0 \\
0 & T & 0 & 0 & 0 & 0 \\
0 & 0 & I & 0 & 0 & 0 \\
0 & 0 & 0 & I & 0 & 0 \\
0 & 0 & 0 & 0 & T & 0 \\
0 & 0 & 0 & 0 & 0 & I
\end{array}\right]<0
$$


and $(4,4)$ blocks in the left side of $(14)$ are $n_{C_{W}}$-dimensional, and the identity matrix in $(6,6)$ block is $(m+p+q)$-dimensional, which are same as those of the identity matrices in (15) and (23).

Remark 5: After the variables $\breve{A}_{f i}, \breve{B}_{f i}, \breve{C}_{f i}$, and $\breve{D}_{f i}$ are obtained from the LMI in (23), we have to perform the decomposition on the matrix $W$ to obtain the solution in (25). Since by most matrix decomposition methods $G_{4}$ and $V$ cannot be determined uniquely, the solution of (25) is not unique.

Remark 6: Theorem 2 presents the conditions under which the residual system is stochastically stable and satisfies the guaranteed performance $\gamma$. The result also covers the case of the perfect communication links existing between the physical plant and the fault detection filter, i.e., there is no data packet dropout and $\bar{\alpha}=1$, with $\Phi_{i j l}$ modified as

$$
\Phi_{i j l}=\left[\begin{array}{cccc}
\Theta_{l} & 0 & \Theta_{15 i j} & \Theta_{16 i j} \\
* & -I & \Theta_{35 i j} & \Theta_{36 i j} \\
* & * & \Theta_{55 i} & 0 \\
* & * & * & -\gamma^{2} I
\end{array}\right]
$$

where $\Theta_{l}, \Theta_{55 i}, \breve{A}_{i}, \breve{B}_{1 i}, \breve{C}_{i}, \breve{D}_{i}$, and $\breve{D}_{W}$ are defined in (24)

$$
\begin{aligned}
\Theta_{15 i j} & =\left[\begin{array}{ll}
U^{T} \breve{A}_{i}+\breve{B}_{f i} \breve{C}_{j} & \breve{A}_{f i} \\
X^{T} \breve{A}_{i}+\breve{B}_{f i} \breve{C}_{j} & \breve{A}_{f i}
\end{array}\right] \\
\Theta_{16 i j} & =\left[\begin{array}{ll}
U^{T} \breve{B}_{1 i}+\breve{B}_{f i} \breve{D}_{j} \\
X^{T} \breve{B}_{1 i}+\breve{B}_{f i} \breve{D}_{j}
\end{array}\right] \\
\Theta_{35 i j} & =\left[\begin{array}{ll}
D_{f i} \breve{C}_{j}-C_{W} & \breve{C}_{f i}
\end{array}\right] \\
\Theta_{36 i j} & =\left[D_{f i} \breve{D}_{j}-\breve{D}_{W}\right] .
\end{aligned}
$$

Remark 7: It is noted that (23) is an LMI over both the matrix variables and the scalar $\gamma$. Among those feasible solutions, the best performance scalar $\gamma$ can be found by solving an optimization problem in which $\gamma$ is included as an optimization variable. The minimum [in terms of the feasibility of (23)] attenuation level of the fault detection filter can be readily obtained by solving the following convex optimization problem using LMI Toolbox: minimize $\gamma$ subject to (23) over $\tilde{P}_{1 l}, \tilde{P}_{2 l}, \tilde{P}_{3 l}, U, X, W, \breve{A}_{f i}, \breve{B}_{f i}, \breve{C}_{f i}$, and $\breve{D}_{f i}$.

\section{FuZZY FAUlt DeteCtion Filter DESIGN FOR UNCERTAIN SYSTEMS}

In this section, the results obtained previously for nominal systems will be extended to fuzzy systems with uncertainties described in (2), i.e., the fuzzy fault detection filter is designed for the uncertain fuzzy system in (4), such that the residual system in (10) is stochastically stable with the performance defined in (11).

Before proceeding further, we first give the following lemma that is needed for our subsequent derivation.

Lemma 1 [31]: Given matrices $\Phi=\Phi^{T}, N, Q$, and $R=$ $R^{T}>0$ of appropriate dimensions

$$
\Phi+N F Q+Q^{T} F^{T} N^{T}<0
$$

for all $F$ satisfying $F^{T} F \leq R$, if and only if there exists a scalar $\varepsilon>0$, such that

$$
\Phi+\varepsilon^{-1} N N^{T}+\varepsilon Q^{T} R Q<0 .
$$

Then, we are in a position to give the fault detection filter design for the fuzzy system with norm bounded uncertainties.

Theorem 3: Consider the fuzzy system in (4). For a given positive constant $\gamma$, if there exist $n$-dimensional matrices

$$
\tilde{P}_{l}=\left[\begin{array}{cc}
\tilde{P}_{1 l} & \tilde{P}_{2 l} \\
\tilde{P}_{2 l}^{T} & \tilde{P}_{3 l}
\end{array}\right]>0
$$

where $n=n_{f}+n_{W}+n_{p}$, matrices $\breve{A}_{f i}, \breve{B}_{f i}, \breve{C}_{f i}$, and $\breve{D}_{f i}$, scalars $\varepsilon_{i j l}$ for any $i, j, l=1, \ldots, r$, and $n$-dimensional matrices $U, X$, and $W$ satisfying the following inequality:

$$
\left[\begin{array}{cc}
\Phi_{i j l}+\varepsilon_{i j l} \tilde{Q}_{i}^{T} \tilde{Q}_{i} & \tilde{N}_{i} \\
\tilde{N}_{i}^{T} & -\varepsilon_{i j l} I
\end{array}\right]<0
$$

where

$$
\begin{aligned}
& \left.\tilde{N}_{i}=\left[\begin{array}{cc}
0 & \breve{N}_{i} \\
0 & 0
\end{array}\right] \quad \breve{N}_{i}=\left[\begin{array}{c}
U^{T} \\
X^{T}
\end{array}\right]\left[\begin{array}{c}
0 \\
N_{i}
\end{array}\right] \quad 0 \quad\left[\begin{array}{c}
0 \\
N_{i}
\end{array}\right]\right] \\
& \tilde{Q}_{i}=\left[\begin{array}{cc}
0 & 0 \\
0 & \breve{Q}_{i}
\end{array}\right] \quad \breve{Q}_{i}=\left[\begin{array}{cccccc}
{\left[\begin{array}{cc}
0 & Q_{a i}
\end{array}\right]} & 0 & 0 & \\
& * & 0 & 0 & \\
& * & * & {\left[Q_{b i}\right.} & 0 & 0
\end{array}\right]
\end{aligned}
$$

and $\Phi_{i j l}$ is defined in Theorem 2, then there exists a fuzzy fault detection filter in the form of (6), such that the residual system is stochastically stable with the performance $\gamma$ defined in (11). Moreover, if the aforementioned condition is satisfied, the matrices for the fault detection filter in (6) are given by

$$
\left[\begin{array}{ll}
A_{f i} & B_{f i} \\
C_{f i} & D_{f i}
\end{array}\right]=\left[\begin{array}{cc}
U^{-T} & 0 \\
0 & I
\end{array}\right]\left[\begin{array}{cc}
\breve{A}_{f i} & \breve{B}_{f i} \\
\breve{C}_{f i} & \breve{D}_{f i}
\end{array}\right]\left[\begin{array}{cc}
V^{-1} & 0 \\
0 & I
\end{array}\right]
$$

with $G_{4}$ and $V$ being nonsingular matrices and satisfying $W=$ $G_{4}^{T} V$.

Proof: Replacing $A_{i}$ and $B_{i}$ in (23) with $A_{i}+N_{i} Z(k) Q_{a i}$ and $B_{i}+N_{i} Z(k) Q_{b i}$, respectively, we have

$$
\Phi_{i j l}+\tilde{N}_{i} \tilde{Z}(k) \tilde{Q}_{i}+\left(\tilde{N}_{i} \tilde{Z}(k) \tilde{Q}_{i}\right)^{T}<0
$$

where $\tilde{Z}(k)$ is an appropriate dimensioned block-diagonal matrix with entries $Z(k)$. According to Lemma 1, the aforementioned inequality holds if

$$
\Phi_{i j l}+\varepsilon_{i j l}^{-1} \tilde{N}_{i} \tilde{N}_{i}^{T}+\varepsilon_{i j l} \tilde{Q}_{i}^{T} \tilde{Q}_{i}<0
$$

which, by Schur complement, is equivalent to the inequality in (31). The proof is completed.

Remark 8: The conditions derived here are based on the basisdependent Lyapunov function method, which can potentially reduce the conservatism of the results. But the computation cost will be increased at the same time, especially when the number of fuzzy rules of the plant is large. One way to solve this problem is to try to reduce the rules in modeling of the physical plant. However, when the number of fuzzy rules for some complex nonlinear systems is large and cannot be reduced in fuzzy modeling, we can adopt the quadratic Lyapunov function approach in solving the fault detection problem. The quadratic approach can be found in [3] and [27].

In this paper, we utilize the flexible and powerful LMI tool to solve the fault detection problem, which is preferred by many researchers [1], [33]. The Schur complement and congruent transformation are used to convert the $H_{\infty}$ norm constraints 
into LMIs feasibility conditions, which are actually convex optimization problems. Solutions can be determined by solving those optimization problems via MATLAB toolbox.

\section{ILLUSTRATIVE EXAMPLES}

In this section, two examples are presented to illustrate the usefulness and applicability of the fault detection filter design approaches developed previously.

\section{A. Example 1}

In this example, we will illustrate the applicability of the fault detection filter design method for the nominal fuzzy system.

Consider a tunnel diode circuit system whose model is established in [8]. With a sampling time $T=0.02 \mathrm{~s}$, the discrete-time model is obtained as

$$
\begin{aligned}
x_{k+1} & =\sum_{i=1}^{2} h_{i}\left(x_{1 k}\right)\left(A_{i} x_{k}+E_{i} w_{k}\right) \\
y_{k} & =\sum_{i=1}^{2} h_{i}\left(x_{1 k}\right)\left(C_{i} x_{k}+F_{i} w_{k}\right)
\end{aligned}
$$

where the state variables are chosen as $x_{1}(t)=v_{C}(t)$ and $x_{2}(t)=i_{L}(t)$, and $v_{C}(t)$ and $i_{L}(t)$ are the capacitor voltage and inductance current, respectively. The parameter matrices are given by

$$
\begin{array}{ll}
A_{1}=\left[\begin{array}{cc}
0.9887 & 0.9024 \\
-0.0180 & 0.8100
\end{array}\right] & E_{1}=\left[\begin{array}{l}
0.0093 \\
0.0181
\end{array}\right] \\
A_{2}=\left[\begin{array}{cc}
0.9033 & 0.8617 \\
-0.0172 & 0.8103
\end{array}\right] & E_{2}=\left[\begin{array}{l}
0.0091 \\
0.0181
\end{array}\right] \\
C_{i}=\left[\begin{array}{ll}
1 & 0
\end{array}\right] & F_{i}=1 .
\end{array}
$$

We assume that there are faults on the capacitor voltage, and the fault matrices are given by

$$
G_{1}=\left[\begin{array}{c}
0.9887 \\
-0.0180
\end{array}\right] \quad G_{2}=\left[\begin{array}{c}
0.9033 \\
-0.0172
\end{array}\right] .
$$

The aim is to design a fuzzy fault detection filter such that the residual system in the form of (13) is stochastically stable and the performance defined in (11) is guaranteed.

The fault weighting system is in the form of (9) and the matrices are chosen as follows:

$$
A_{W}=0.1 \quad B_{W}=0.25 \quad C_{W}=0.5 \quad D_{W}=0 .
$$

We first consider the perfect communication case, i.e., there is no data packet dropout between the physical plant and the fault detection filter, and thus, $\bar{\alpha}=1$. By solving the LMI in Theorem 2 , the matrix variables are obtained as

$$
\begin{aligned}
& \breve{A}_{f 1}=\left[\begin{array}{ccc}
0.0301 & -0.0043 & -0.0628 \\
-0.0028 & 0.0010 & 0.0273 \\
-0.0038 & 0.0210 & 0.7217
\end{array}\right] \quad \breve{D}_{f 1}=0.0369 \\
& \breve{B}_{f 1}=\left[\begin{array}{c}
0.0164 \\
-0.0014 \\
0.0063
\end{array}\right] \quad \breve{C}_{f 1}=\left[\begin{array}{lll}
-0.5023 & 0.0372 & 0.0066
\end{array}\right]
\end{aligned}
$$

$$
\begin{aligned}
\breve{A}_{f 2}= & {\left[\begin{array}{ccc}
0.0294 & -0.0048 & -0.0658 \\
-0.0027 & 0.0011 & 0.0273 \\
-0.0039 & 0.0222 & 0.7146
\end{array}\right] \quad \breve{D}_{f 2}=0.0368 } \\
\breve{B}_{f 2}= & {\left[\begin{array}{c}
0.0143 \\
-0.0011 \\
-0.0104
\end{array}\right] \quad \breve{C}_{f 2}=\left[\begin{array}{lll}
-0.4995 & 0.0372 & 0.0041
\end{array}\right] } \\
W= & {\left[\begin{array}{ccc}
0.2959 & -0.0250 & -0.0736 \\
-0.0248 & 0.0033 & 0.0327 \\
-0.0778 & 0.0330 & 0.8847
\end{array}\right] }
\end{aligned}
$$

and the guaranteed performance defined in (11) is $\gamma^{*}=0.1042$. Applying a full-rank factorization on $W$, we get $G_{4}$ and $V$

$$
\begin{gathered}
G_{4}=\left[\begin{array}{ccc}
0.1249 & -0.0399 & -0.9914 \\
0.9893 & -0.0711 & 0.1275 \\
-0.0755 & -0.9967 & 0.0306
\end{array}\right] \\
V=\left[\begin{array}{ccc}
0.1151 & -0.0360 & -0.8876 \\
0.2845 & -0.0207 & 0.0377 \\
-0.0000 & -0.0004 & 0.0000
\end{array}\right]
\end{gathered}
$$

and thus, the fuzzy fault detection filter matrices can be calculated by (25)

$$
\begin{aligned}
& A_{f 1}=\left[\begin{array}{ccc}
0.8034 & -0.2985 & -3.1752 \\
-0.0266 & 0.1147 & 0.6210 \\
0.0005 & 0.0011 & 0.0089
\end{array}\right] \quad D_{f 1}=0.0369 \\
& B_{f 1}=\left[\begin{array}{c}
-0.0042 \\
0.0171 \\
0.0004
\end{array}\right] \quad C_{f 1}=\left[\begin{array}{lll}
-0.0810 & -1.7323 & 4.1194
\end{array}\right] \\
& A_{f 2}=\left[\begin{array}{ccc}
0.7961 & -0.2950 & 0.3848 \\
-0.0224 & 0.1104 & 0.1722 \\
0.0005 & 0.0009 & 0.0245
\end{array}\right] \quad D_{f 2}=0.0368
\end{aligned}
$$$$
B_{f 2}=\left[\begin{array}{c}
-0.0085 \\
0.0156 \\
0.0003
\end{array}\right] \quad C_{f 2}=\left[\begin{array}{lll}
-0.0779 & -1.7237 & 3.4360
\end{array}\right] \text {. }
$$

Fig. 1 shows the residual response $r_{k}$ and the response of the residual evaluation function $\|\cdot\|_{T}$ varying as time $k$ when $w_{k}=$ 0 , where the fault is supposed to be

$$
f_{k}= \begin{cases}1, & 300 \leq k \leq 600 \\ 0, & \text { else. }\end{cases}
$$

From the figure, we can see that the designed filter can detect the fault effectively when it occurs.

Then, we assume the disturbance

$$
w_{k}= \begin{cases}\operatorname{rand}[0,1], & 200 \leq k \leq 700 \\ 0, & \text { else. }\end{cases}
$$

Fig. 2 shows the residual response and the residual evaluation function response with the disturbance $w_{k}$, respectively, which indicate that the residual can not only reflect the fault in time, but also recognize the fault without confusing it with the disturbance $w_{k}$.

Next, we consider the fault detection problem with imperfect communication links. The parameter is assumed to be $\bar{\alpha}=0.8$, which means that there are $20 \%$ data lost during the transmission 

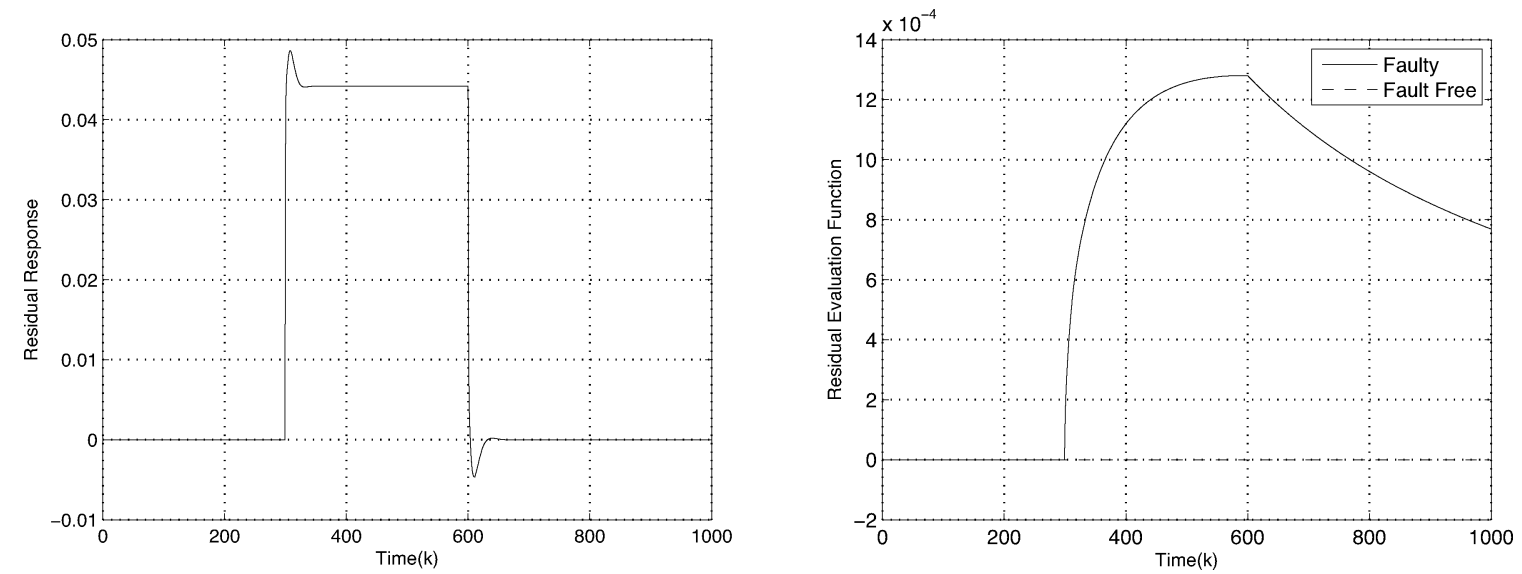

Fig. 1. Residual response and evaluation function for the nominal system with zero $u_{k}$ and $w_{k}(\bar{\alpha}=1)$.
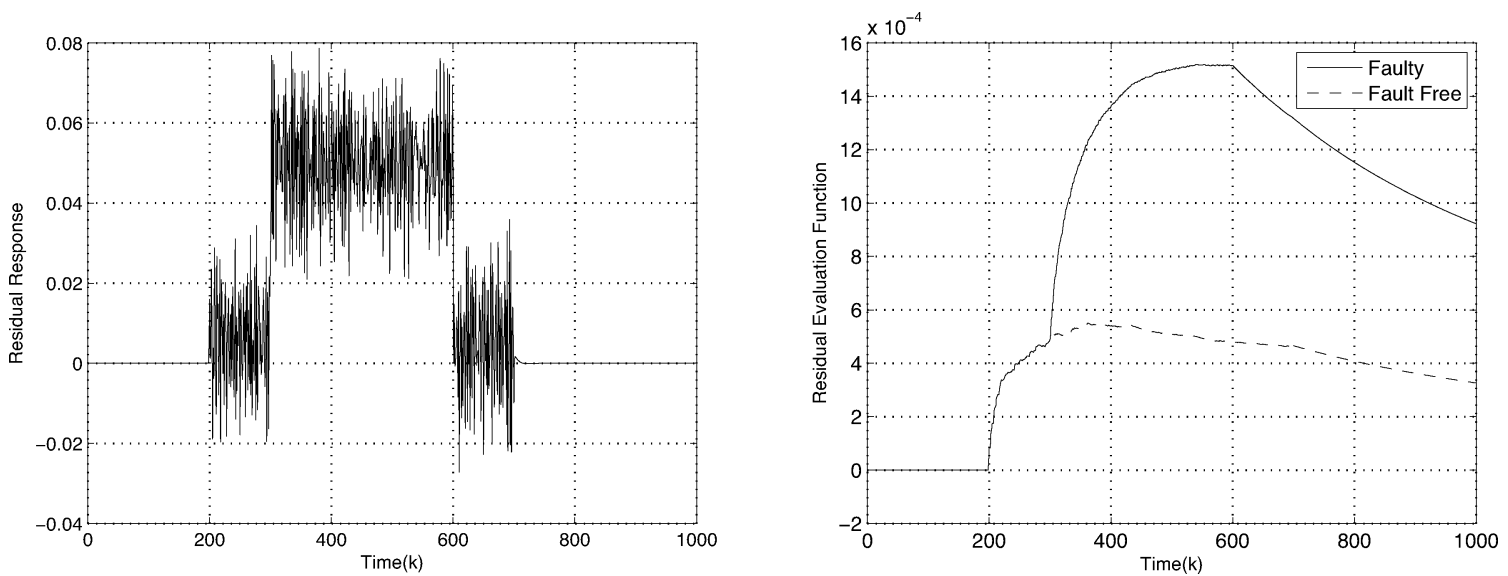

Fig. 2. Residual response and evaluation function for the nominal system with $w_{k}(\bar{\alpha}=1)$.

from the physical plant to the fault detection filter. By applying Theorem 2, the fuzzy fault detection filter matrices are given by

$$
\begin{aligned}
& A_{f 1}=\left[\begin{array}{ccc}
0.7880 & -0.4444 & 10.9624 \\
-0.0734 & 0.1514 & -8.8659 \\
-0.0007 & -0.0010 & 0.6085
\end{array}\right] \quad D_{f 1}=0.0240 \\
& B_{f 1}=\left[\begin{array}{c}
0.0007 \\
-0.0046 \\
0.0002
\end{array}\right] \quad C_{f 1}=\left[\begin{array}{lll}
0.1412 & 1.7282 & 11.2867
\end{array}\right] \\
& A_{f 2}=\left[\begin{array}{ccc}
0.7986 & -0.4315 & 8.3850 \\
-0.0754 & 0.1482 & -5.3386 \\
-0.0005 & -0.0008 & 0.3067
\end{array}\right] \quad D_{f 2}=0.0240 \\
& B_{f 2}=\left[\begin{array}{c}
0.0022 \\
-0.0049 \\
0.0003
\end{array}\right] \quad C_{f 2}=\left[\begin{array}{lll}
0.1560 & 1.7534 & 7.8455
\end{array}\right]
\end{aligned}
$$

and the guaranteed performance is $\gamma^{*}=0.1216$. By assuming the same fault as in (34) and zeros disturbance $w_{k}$, Fig. 3 shows the residual response of $r_{k}$ and the evaluation function response, which clearly tell us that the fault can be detected effectively when it appears.

Then, assume the same disturbance as that in (35). Fig. 4 shows the residual response and the evaluation function re- sponse, which clearly indicate that the residual can also detect the fault without confusing it with the disturbance.

It is worth noting that the obtained minimum-guaranteed performance $\gamma^{*}$ will change as the different values of $\bar{\alpha}$, which is shown in Table I. From the table, we know that as the values of $\bar{\alpha}$ become larger, the higher $\gamma^{*}$ can be obtained. This is true since the larger $\bar{\alpha}$ means the less missing measurements; therefore, the better disturbance attenuation performance $\gamma^{*}$ can be obtained.

\section{B. Example 2}

In this example, we will design a fuzzy fault detection filter for the fuzzy system with uncertainties. We still consider the fuzzy system in Example 1. For simulation, we assume there are some uncertainties in the form of (2), and the parameters are given by

$$
N_{i}=\left[\begin{array}{l}
0.25 \\
0.25
\end{array}\right] \quad Q_{a i}=\left[\begin{array}{ll}
0.1 & 0
\end{array}\right] \quad Q_{b i}=0.1
$$

First, we consider the perfect communication condition $(\bar{\alpha}=$ 1). By solving the inequalities in Theorem 3, the minimum performance value is obtained as $\gamma^{*}=0.1043$, and the fuzzy 

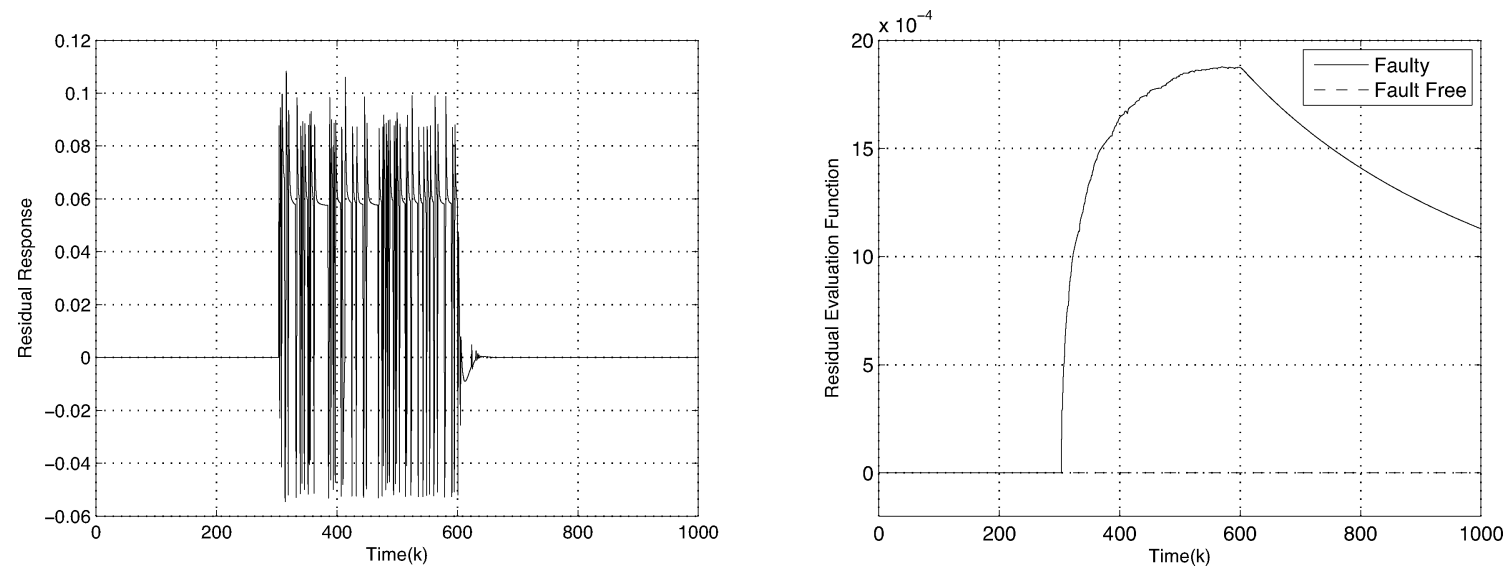

Fig. 3. Residual response and evaluation function for the nominal system with zero $u_{k}$ and $w_{k}(\bar{\alpha}=0.8)$.
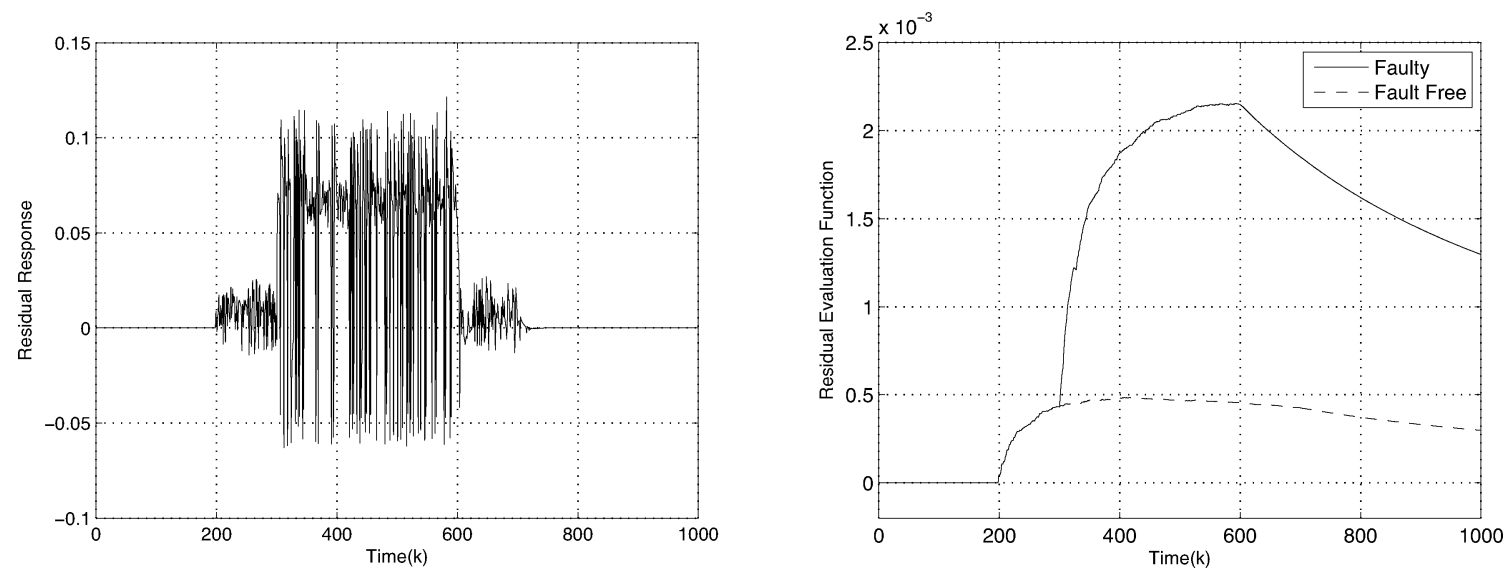

Fig. 4. Residual response and evaluation function for the nominal system with $w_{k}(\bar{\alpha}=0.8)$.

TABLE I

Minimum GuARANTEED PERFORMANCE $\gamma$ FOR DIFFERENT VALUES OF $\bar{\alpha}$

\begin{tabular}{ccccccc}
\hline $\bar{\alpha}$ & 0.7 & 0.75 & 0.8 & 0.85 & 0.9 & 1 \\
\hline$\gamma^{*}$ & 0.1258 & 0.1239 & 0.1216 & 0.1184 & 0.1139 & 0.1042 \\
\hline
\end{tabular}

detection filter matrices are

$$
\begin{aligned}
& A_{f 1}=\left[\begin{array}{ccc}
0.7912 & -0.2836 & 1.5684 \\
-0.1096 & 0.1483 & -0.7187 \\
-0.0013 & -0.0008 & -0.0020
\end{array}\right] \quad D_{f 1}=0.0377 \\
& B_{f 1}=\left[\begin{array}{c}
-0.0017 \\
-0.0166 \\
0.0003
\end{array}\right] \quad C_{f 1}=\left[\begin{array}{lll}
0.3017 & 1.6957 & 4.1770
\end{array}\right] \\
& A_{f 2}=\left[\begin{array}{ccc}
0.7660 & -0.2853 & -0.9327 \\
-0.1011 & 0.1451 & -0.9949 \\
-0.0013 & -0.0005 & 0.0236
\end{array}\right] \quad D_{f 2}=0.0376 \\
& B_{f 2}=\left[\begin{array}{c}
0.0014 \\
-0.0154 \\
0.0003
\end{array}\right] \quad C_{f 2}=\left[\begin{array}{lll}
0.2969 & 1.6880 & 3.5792
\end{array}\right] \text {. }
\end{aligned}
$$

Next, we consider the robust case with intermittent measurements. Without loss of generality, we suppose $\bar{\alpha}=0.8$. The minimum performance defined in (11) is obtained as $\gamma^{*}=0.1241$, and the fuzzy fault detection filter matrices are obtained as

$$
\begin{aligned}
& A_{f 1}=\left[\begin{array}{ccc}
0.2766 & -0.3567 & -5.0519 \\
-0.2921 & 0.6742 & -6.2744 \\
-0.0018 & 0.0009 & 0.6778
\end{array}\right] \quad D_{f 1}=0.0203 \\
& C_{f 1}=\left[\begin{array}{lll}
1.4222 & 1.0240 & 10.7865
\end{array}\right] \quad B_{f 1}=\left[\begin{array}{c}
-0.0017 \\
-0.0029 \\
0.0002
\end{array}\right] \\
& A_{f 2}=\left[\begin{array}{ccc}
0.2961 & -0.3280 & -1.9103 \\
-0.2847 & 0.6502 & -6.2402 \\
-0.0013 & -0.0003 & 0.4207
\end{array}\right] \quad D_{f 2}=0.0195 \\
& B_{f 2}=\left[\begin{array}{c}
-0.0011 \\
-0.0037 \\
0.0002
\end{array}\right] \quad C_{f 2}=\left[\begin{array}{lll}
1.4503 & 0.9920 & 8.0067
\end{array}\right] .
\end{aligned}
$$

Fig. 5 shows the residual response and the response of the residual evaluation function. It can be seen that the designed fuzzy 

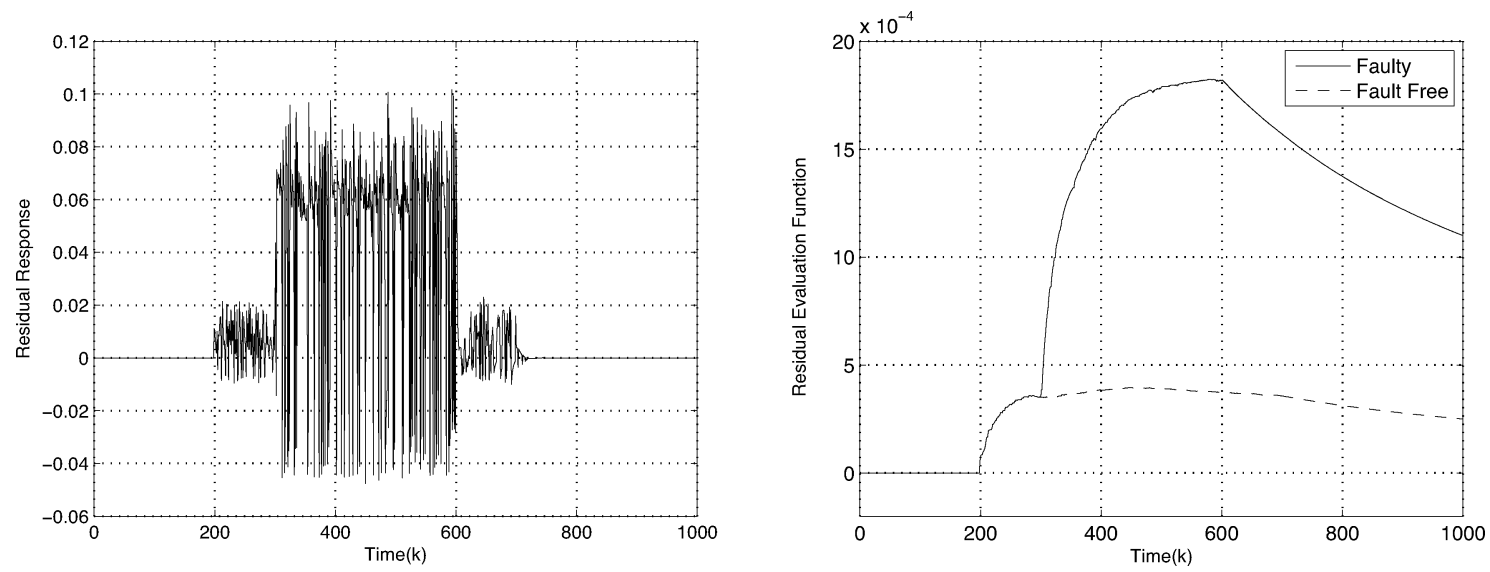

Fig. 5. Residual response and evaluation function for the uncertain system with $w_{k}(\bar{\alpha}=0.8)$.

fault detection filter is also effective for the uncertain fuzzy system with missing measurements.

\section{CONCLUDING REMARKS}

In this paper, the problem of fault detection for T-S fuzzy systems with intermittent measurements has been investigated. The communication links between the plant and the fault detection filter are assumed to be imperfect, and a stochastic variable satisfying the Bernoulli random binary distribution is utilized to model the unreliable communication links. A fuzzy fault detection filter has been designed such that, for all data missing conditions, the residual system is stochastically stable and preserves a guaranteed performance. The results have been extended to the T-S fuzzy systems with time-varying parameter uncertainties. All the results are formulated in the form of LMIs. Two examples have been provided to illustrate the usefulness and applicability of the results.

\section{REFERENCES}

[1] A. Casavola, D. Famularo, and G. Franzè, "A robust deconvolution scheme for fault detection and isolation of uncertain linear systems: An LMI approach," Automatica, vol. 41, no. 8, pp. 1463-1472, 2005.

[2] A. Fekih, H. Xu, and F. Chowdhury, "Neural networks based system identification techniques for model based fault detection of nonlinear systems," Int. J. Innov.. Comput., Inf. Control, vol. 3, no. 5, pp. 1073-1085, 2007.

[3] G. Feng, "A survey on analysis and design of model-based fuzzy control systems," IEEE Trans. Fuzzy Syst., vol. 14, no. 5, pp. 676-697, Oct. 2006.

[4] P. Frank and X. Ding, "Frequency domain approach to optimally robust residual generation and evaluation for model-based fault diagnosis," $\mathrm{Au}$ tomatica, vol. 30, pp. 789-804, 1994.

[5] P. Frank and X. Ding, "Survey of robust residual generation and evaluation methods in observer-based fault detection systems," J. Process Control, vol. 7, pp. 403-424, 1997.

[6] H. Gao and C. Wang, "Delay-dependent robust $H_{\infty}$ and $L_{2}-L_{\infty}$ filtering for a class of uncertain nonlinear time-delay systems," IEEE Trans. Autom. Control, vol. 48, no. 9, pp. 1661-1666, Sep. 2003.

[7] H. Gao and C. Wang, "Robust $L_{2}-L_{\infty}$ filtering for uncertain systems with multiple time-varying state delays," IEEE Trans. Circuits Syst. I, Fundam. Theory Appl., vol. 50, no. 4, pp. 594-599, Apr. 2003.

[8] W. Assawinchaichote and S. K. Nguang, " $H_{\infty}$ filtering for fuzzy dynamic systems with D stability constraints," IEEE Trans. Circuits Syst. I, Fundam. Theory Appl., vol. 50, no. 11, pp. 1503-1508, Nov. 2003.

[9] I. Izadi, T. Chen, and Q. Zhao, "Norm invariant discretization for sampleddata fault detection," Automatica, vol. 41, no. 9, pp. 1633-1637, 2005.
[10] B. Jiang, M. Staroswiecki, and V. Cocquempot, " $H_{\infty}$ fault detection filter design for linear discrete-time systems with multiple time delays," Int. $J$. Syst. Sci., vol. 34, no. 5, pp. 365-373, 2003.

[11] M. Bask, A. Johansson, and T. Norlander, "Dynamic threshold generators for robust fault detection in linear systems with parameter uncertainty," Automatica, vol. 42, pp. 1095-1106, 2006.

[12] J. Lam and S. S. Zhou, "Dynamic output feedback $H_{\infty}$ control of discretetime fuzzy systems: A fuzzy-basis-dependent Lyapunov function approach," Int. J. Syst. Sci., vol. 38, no. 1, pp. 25-37, 2007.

[13] C. Lin, Q. G. Wang, and T. H. Lee, "Stability and stabilization of a class of fuzzy time-delay descriptor systems," IEEE Trans. Fuzzy Syst., vol. 14 no. 4, pp. 542-551, Aug. 2006.

[14] Z. Mao, B. Jiang, and P. Shi, " $H_{\infty}$ fault detection filter design for networked control systems modelled by discrete Markovian jump systems," IET Control Theory Appl., vol. 1, no. 5, pp. 1336-1343, 2007.

[15] Z. Mao and B. Jiang, "Fault identification and fault-tolerant control for a class of network control systems," Int. J. Innov. Comput., Inf. Control, vol. 3, no. 5, pp. 1121-1130, 2007.

[16] R. Mattone and A. D. Luca, "Relaxed fault detection and isolation: An application to a nonlinear case study," Automatica, vol. 42, no. 1, pp. 109116,2006

[17] S. K. Nguang, P. Shi, and S. Ding, "Fault detection for uncertain fuzzy systems: An LMI approach," IEEE Trans. Fuzzy Syst., vol. 15, no. 6 , pp. 1251-1262, Dec. 2007.

[18] D. C. Oh and J. H. Kim, "A simple frequency weighted model reduction using structurally balanced truncation: Existence of solutions," Int. J. Control, vol. 75, pp. 1190-1195, 2002.

[19] P. Shi, "Filtering on sampled-data systems with parametric uncertainty," IEEE Trans. Autom. Control, vol. 43, no. 7, pp. 1022-1027, Jul. 1998.

[20] P. Shi, E. K. Boukas, and R. K. Agarwal, "Kalman filtering for continuoustime uncertain systems with Markovian jumping parameters," IEEE Trans. Autom. Control, vol. 44, no. 8, pp. 1592-1597, Aug. 1999.

[21] P. Shi and S. K. Nguang, " $H_{\infty}$ output feedback control of fuzzy system models under sampled measurements," Comput. Math. Appl., vol. 46, no. 5-6, pp. 705-717, 2003.

[22] P. Shi, Y. Xia, G. P. Liu, and D. Rees, "On designing of sliding-mode control for stochastic jump systems," IEEE Trans. Autom. Control, vol. 51, no. 1, pp. 97-103, Jan. 2006.

[23] I. Skrjanc, S. Blazic, and O. Agamennoni, "Identification of dynamical systems with a robust interval fuzzy model," Automatica, vol. 41, pp. 327$332,2005$.

[24] A. Stoorvogel, H. Niemann, A. Saberi, and P. Sannuti, "Optimal fault signal estimation," Int. J. Robust Nonlinear Control, vol. 12, pp. 697727, 2002.

[25] H. Wang and J. Lam, "Robust fault detection for uncertain discrete-time systems," J. Guid. Control Dyn., vol. 25, no. 2, pp. 291-301, 2002.

[26] H. Wang, J. Wang, and J. Lam, "Robust fault detection observer design: Iterative LMI approaches," J. Dyn. Syst. Meas. Control, vol. 129, no. 1, pp. $77-82,2007$.

[27] H. O. Wang, K. Tanaka, and M. F. Griffin, "An approach to fuzzy control of nonlinear systems: Stability and design issues," IEEE Trans. Fuzzy Syst., vol. 4, no. 2, pp. 14-23, Feb. 1996. 
[28] Z. Wang, D. W. C. Ho, and X. Liu, "Variance-constrained filtering for uncertain stochastic systems with missing measurements," IEEE Trans. Autom. Control, vol. 48, no. 7, pp. 1254-1258, Jul. 2003.

[29] Z. Wang, F. Yang, D. W. C. Ho, and X. Liu, "Robust finite-horizon filtering for stochastic systems with missing measurements," IEEE Signal Process. Lett., vol. 12, no. 6, pp. 437-440, Jun. 2005.

[30] A. S. Willsky and H. L. Jones, "A generalized likelihood ratio approach to the detection and estimation of jumps in linear sytems," IEEE Trans. Autom. Control, vol. AC-21, no. 1, pp. 108-112, Feb. 1976.

[31] L. Xie, "Output feedback $H_{\infty}$ control of systems with parameter uncertainty," Int. J. Control, vol. 63, pp. 741-750, 1996.

[32] P. Zhang, S. X. Ding, G. Z. Wang, and D. H. Zhou, "A frequency domain approach to fault detection in sampled-data systems," Automatica, vol. 39, no. 7, pp. 1303-1307, 2003.

[33] M. Zhong, S. X. Ding, J. Lam, and H. Wang, "An LMI approach to design robust fault detection filter for uncertain LTI systems," $A u$ tomatica, vol. 39, no. 3, pp. 543-550, 2003.

[34] S. Zhou, G. Feng, J. Lam, and S. Xu, "Robust $H_{\infty}$ control for discretetime fuzzy systems via basis-dependent Lyapunov functions," Inf. Sci., vol. 174, no. 3/4, pp. 197-217, 2005.

[35] S. Xu and J. Lam, "Exponential $H_{\infty}$ filter design for uncertain TakagiSugeno fuzzy systems with time delay," Eng. Appl. Artif. Intell., vol. 17, pp. 645-659, 2004.

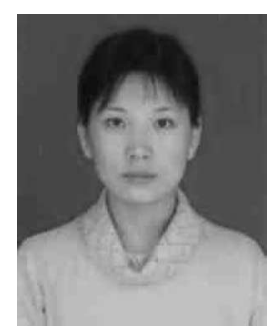

Yan Zhao received the B.S. degree in chemical engineering and equipment control and the M.S. degree in mechanical engineering from the Inner Mongolia University of Technology, Hohhot, China, in 2002 and 2005 , respectively. She is currently working toward the $\mathrm{Ph} . \mathrm{D}$. degree in control science and engineering at Harbin Institute of Technology, Harbin, China.

Her current research interests include fuzzy control systems, robust control, and networked control systems.

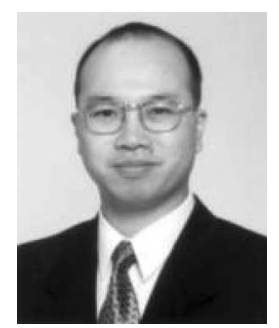

James Lam (S'86-M'86-SM'99) received the B.Sc. degree (with first class) in mechanical engineering from the University of Manchester, Manchester, U.K., and the M.Phil. and Ph.D. degrees in control engineering from the University of Cambridge, Cambridge, U.K. He received the Ashbury Scholarship, the A.H. Gibson Prize, and the H. Wright Baker Prize for his academic performance.

$\mathrm{He}$ is currently a Professor in the Department of Mechanical Engineering, University of Hong Kong, Hong Kong. He is an Associate Editor of the Asian Journal of Control, International Journal of Systems Science, Journal of Sound and Vibration, International Journal of Applied Mathematics and Computer Science, the IEEE TRANSACTIONS ON SIGNAL PROCESSING, Journal of the Franklin Institute, Dynamics of Continuous, Discrete and Impulsive Systems (Series B: Applications and Algorithms), and Automatica. He is also a member of the Editorial Board of the Institution of Engineering and Technology (IET) Control Theory and Applications, Open Electrical and Electronic Engineering Journal, Research Letters in Signal Processing, International Journal of Systems, Control and Communications, and Journal of Electrical and Computer Engineering. His current research interests include reduced-order modeling, delay systems, descriptor systems, stochastic systems, multidimensional systems, robust control, and filtering. He was an Editor-in-Chief of the Institution of Electrical Engineers (IEE) Proceedings Control Theory and Applications.

Prof. Lam is a Chartered Mathematician and a Chartered Scientist. He is a Fellow of the Institute of Mathematics and Its Applications, and the IET. He is a Scholar and a Fellow of the Croucher Foundation.

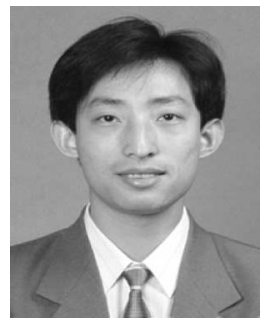

Huijun Gao (M'06) was born in Heilongjiang, China, in 1976. He received the M.S. degree in electrical engineering from Shenyang University of Technology, Shenyang, China, in 2001, and the Ph.D. degree in control science and engineering from Harbin Institute of Technology, Harbin, China, in 2005.

From November 2003 to August 2004, he was a Research Associate in the Department of Mechanical Engineering, University of Hong Kong, Hong Kong. In November 2004, he joined Harbin Institute of Technology, where he is currently a Professor. From October 2005 to October 2007, he was a Postdoctoral Researcher in the Department of Electrical and Computer Engineering, University of Alberta, Edmonton, $\mathrm{AB}, \mathrm{Canada}$. He is an Associate Editor of the Journal of Intelligent and Robotics Systems, Circuits, System and Signal Processing, etc. His current research interests include network-based control, robust control, and time-delay systems and their industrial applications.

Prof. Gao is an Associate Editor of the IEEE TRANSACTIONS ON SySTEMS, MAN AND CYBERNETICS PART B: CYBERNETICS and the IEEE TRANSACTIONS ON INDUSTRIAL ELECTRONICS. He was an outstanding reviewer for the IEEE TranSACTIONS ON AUTOMATIC CONTROL and Automica in 2008 and 2007, respectively, and an appreciated reviewer for the IEEE TRANSACTIONS ON SIGNAL PROCESSING in 2006. He was the recipient of the University of Alberta Dorothy J. Killam Memorial Postdoctoral Fellow Prize in 2005, the National Outstanding Youth Science Fund in 2008, and the National Outstanding Doctoral Thesis Award in 2007. He was the corecipient of the National Natural Science Award of China in 2008. 